\title{
Responses to acute infection with SARS-CoV-2 in the lungs of rhesus macaques, baboons and
}

\section{marmosets}

\author{
Dhiraj Kumar Singh ${ }^{1,2}$, Bindu Singh ${ }^{1,2}$, Shashank R. Ganatra ${ }^{1,2}$, Michal Gazi ${ }^{2}$, Journey Cole ${ }^{1,2}$, \\ Rajesh Thippeshappa ${ }^{1,2}$, Kendra J. Alfson ${ }^{2}$, Elizabeth Clemmons ${ }^{1,2}$, Olga Gonzalez ${ }^{1,2}$, Ruby Escobedo ${ }^{1,2}$, \\ Tae-Hyung Lee ${ }^{1,2}$, Ayan Chatterjee ${ }^{1,2}$, Yenny Goez-Gazi' ${ }^{2}$, Riti Sharan ${ }^{1,2}$, Maya Gough ${ }^{1,2}$, \\ Cynthia Alvarez ${ }^{1,2}$, Alyssa Blakley ${ }^{1,2}$, Justin Ferdin ${ }^{1,2}$, Carmen Bartley ${ }^{1,2}$, Hilary Staples ${ }^{1,2}$, \\ Laura Parodi 1,2, Jessica Callery 1,2, Amanda Mannino, ${ }^{1,2}$, Benjamin Klaffke'2, Priscilla Escareno2, \\ Roy N. Platt II@2, Vida Hodara ${ }^{1,2}$, Julia Scordo², Shalini Gautam², Andreu G. Vilanova $\circledast^{2}$, \\ Angelica Olmo-Fontanez ${ }^{2}$, Alyssa Schami ${ }^{2}$, Adelekan Oyejide ${ }^{3}$, Dharani K. Ajithdoss ${ }^{3}{ }^{3}$, \\ Richard Copin $\oplus^{3}$, Alina Baum ${ }^{3}$, Christos Kyratsous ${ }^{3}$, Xavier Alvarez ${ }^{1,2}$, Mushtaq Ahmed ${ }^{4}$, \\ Bruce Rosa ${ }^{4}{ }^{4}$, Anna Goodroe ${ }^{1,2}$, John Dutton ${ }^{1,2}$, Shannan Hall-Ursone ${ }^{1,2}$, Patrice A. Frost ${ }^{1,2}$, \\ Andra K. Voges ${ }^{1,2,5}$, Corinna N. Ross ${ }^{1,2}$, Ken Sayers ${ }^{1,2}$, Christopher Chen ${ }^{10,2}$, Cory Hallam ${ }^{2}$, \\ Shabaana A. Khader ${ }^{4}$, Makedonka Mitreva ${ }^{4}$, Timothy J. C. Anderson'2, Luis Martinez-Sobrido²,

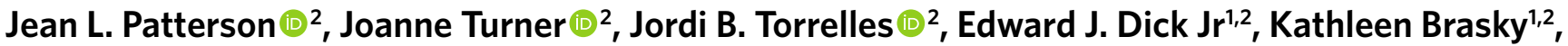

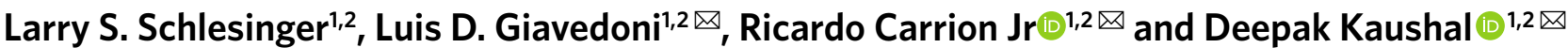

Non-human primate models will expedite therapeutics and vaccines for coronavirus disease 2019 (COVID-19) to clinical trials. Here, we compare acute severe acute respiratory syndrome coronavirus 2 (SARS-CoV-2) infection in young and old rhesus macaques, baboons and old marmosets. Macaques had clinical signs of viral infection, mild to moderate pneumonitis and extra-pulmonary pathologies, and both age groups recovered in two weeks. Baboons had prolonged viral RNA shedding and substantially more lung inflammation compared with macaques. Inflammation in bronchoalveolar lavage was increased in old versus young baboons. Using techniques including computed tomography imaging, immunophenotyping, and alveolar/peripheral cytokine response and immunohistochemical analyses, we delineated cellular immune responses to SARS-CoV-2 infection in macaque and baboon lungs, including innate and adaptive immune cells and a prominent type-I interferon response. Macaques developed T-cell memory phenotypes/responses and bystander cytokine production. Old macaques had lower titres of SARS-CoV-2-specific IgG antibody levels compared with young macaques. Acute respiratory distress in macaques and baboons recapitulates the progression of COVID-19 in humans, making them suitable as models to test vaccines and therapies.

E arly reports indicate that non-human primates (NHPs) are suitable models for the pathology of severe acute respiratory syndrome coronavirus 2 (SARS-CoV-2) infection/coronavirus disease 2019 (COVID-19), and for evaluating vaccine candidates $^{1-6}$. These studies delineated the associated histopathology and morbidity at terminal end points for rhesus macaques, cynomolgus macaques and African green monkeys. To further expand the existing understanding of the NHP models of SARS-CoV-2 infection $^{1-6}$, we also evaluated baboon and old marmoset models. Furthermore, we expanded on the rhesus macaque model of SARS-CoV-2 infection using computed tomography (CT) imaging and flow-cytometry-based cellular phenotyping, as well as by analysing alveolar and peripheral cytokine responses during the course of the disease development and resolution. We also complemented the data with matched immunological and histopathological findings in organs from the terminal end points. We sought to characterize early cellular immune events in the lungs after SARS-CoV-2 infection in three NHP genera: Indian rhesus macaques (old and young), baboons (old and young) and common marmosets (old). In the early stages, macaques developed clinical signatures of viral infection and systemic inflammation, early evidence of viral replication, mild to moderate interstitial and alveolar pneumonitis, and moderate progressive pneumonia. In longitudinal studies over two weeks, young and old macaques showed early signs of COVID-19 with recovery in both age groups. Analysis of blood and bronchoalveolar lavage (BAL) revealed a complex early inflammatory milieu with influx in the lungs of innate and adaptive immune cells, particularly myeloid cells, and a prominent type-I interferon (IFN)

'Southwest National Primate Research Center, San Antonio, TX, USA. ${ }^{2}$ Texas Biomedical Research Institute, San Antonio, TX, USA. ${ }^{3}$ Regeneron Pharmaceuticals Inc., Tarrytown, NY, USA. ${ }^{4}$ Washington University School of Medicine in St Louis, St Louis, MO, USA. ${ }^{5}$ Veterinary Imaging Consulting of South Texas, San Antonio, TX, USA.凶e-mail: Igiavedoni@txbiomed.org; rcarrion@txbiomed.org; dkaushal@txbiomed.org 
response. Whereas rhesus macaques exhibited moderate disease, baboons were susceptible to SARS-CoV-2 infection with extensive pathology after infection, and marmosets demonstrated mild infection. Thus, different NHP species exhibit heterogeneous responses to SARS-CoV-2 infection. Rhesus macaques and baboons develop different, quantifiable disease attributes making them essential models to test vaccines and therapeutics against COVID-19.

\section{Results}

Heterogeneity in SARS-CoV-2 viral loads in young and old NHPs. Macaques, baboons and old marmosets were infected by multiple routes (ocular, intratracheal and intranasal) with sixth-passage, fully sequenced and authenticated virus at a target dose of $1.05 \times 10^{6}$ plaque-forming units (p.f.u.) per animal. SARS-CoV-2 viral RNA (vRNA) was detected early in all species at $3 \mathrm{~d}$ post-infection (d.p.i.; Fig. 1a-o and Supplementary Table 1) and decreased thereafter at variable rates. Comparable BAL vRNA levels were detected in young and old macaques at 3 d.p.i. (5/6 each; Fig. 1a). Almost no BAL vRNA was detected at 9 d.p.i. (1/12) and none was detected at 12 d.p.i. (Fig. 1a). vRNA in nasopharyngeal swabs (NS) could be detected in $50 \%$ of animals at 3 d.p.i., $10 / 12$ ( 6 young, 4 old) at 9 d.p.i. and 6/12 at the study end (Fig. 1b). vRNA was detected in $4 / 12$ and 2/12 animals, respectively, from rectal swabs (RS; Fig. 1c) and in buccopharyngeal swabs (BS; Extended Data Fig. 1a) at 3 and 6 d.p.i., but infrequently at later time points. vRNA was detected in the lungs of 8/12 (3 young, 5 old) macaques at necropsy (14-17 d.p.i.; Fig. 1d). No vRNA was detected in any plasma samples (Extended Data Fig. 1b) or in randomly selected urine samples (Extended Data Fig. 1c). We also detected no SARS-CoV-2 subgenomic RNA (a correlate for infectious/replicating virus) in either rhesus (Extended Data Fig. 1d) or baboon (Extended Data Fig. 1e) lungs in the longitudinal study (Fig. 1m). Thus, despite vRNA persistence in the lungs of immunocompetent macaques, the absence of replicative virus indicates that macaques control SARS-CoV-2 infection.

The pattern of vRNA in baboons mimicked that of macaques (Fig. 1e-h). At 3 d.p.i., 8/12 and 10/12 baboons had detectable vRNA in BAL (Fig. 1e) and NS (Fig. 1f), respectively. A comparatively higher number of old baboons (4/6) harboured BAL vRNA at 9 d.p.i. relative to young baboons (0/6) (Fig. 1e). Despite these differences, peak vRNA in BAL $(\sim 4 \log )$ was detected in both age groups at 3 d.p.i. (Fig. 1e). vRNA levels detected in NS (Fig. 1f) and RS (Fig. 1g) of old baboons were higher compared with swabs from young baboons. Peak vRNA levels were detected at 3 d.p.i. (6/6 old and 4/6 young baboons) in NS. At 6 and 9d.p.i., 4/6 old (mean $>3 \log$ ) and only $1 / 6$ young (mean $<1 \log$ ) baboons, respectively, were positive for vRNA in NS (Fig. 1f). Peak vRNA was detected at 9 d.p.i. for both age groups of baboons in RS (Fig. 1g), with an average of $\sim 5 \log$ in old baboons, which was higher than in young baboons $(\sim 3 \mathrm{log}$; Fig. $1 \mathrm{~g})$. vRNA was also detected in the lungs of five old baboons at necropsy (14-17 d.p.i.; Fig. 1h) but no SARS-CoV-2 subgenomic RNA was detected (Fig. 1n and Extended Data Fig. 1e). Although no statistically significant differences were observed for BAL vRNA levels (Fig. 1i) and NS vRNA levels (Fig. 1j) between baboons and macaques, RS showed substantial differences; baboons harboured greater levels (by several log) throughout the infection protocol (Fig. 1k). Differences in lung viral titres were not significantly different between rhesus and baboons (Fig. 11).

Less than $4 \log$ of vRNA was detected in NS from infected old marmosets, peaking at 3 d.p.i., and 1/6 animals was also positive at 6 d.p.i. (Fig. 1o). No vRNA was detected in BS throughout the study (Fig. 1p). In comparison to macaques and baboons, marmosets had accelerated clearance of vRNA. Only low lung vRNA levels were detected at study end (Fig. 1q).

Gross examination at necropsy (14-17 d.p.i.) identified red discoloration of the lung lobes in 50\% of macaques (Extended Data Fig. 2b) and $100 \%$ of baboons (Extended Data Fig. 3a,b). Supplementary Tables 2 (macaques) and 3 (baboons) summarize the histopathological findings. The lungs were the most affected organ in each case (Extended Data Figs. 2a-d and 3, and Supplementary Tables 2 and 3). Multifocal minimal to mild interstitial mononuclear inflammation was seen in 11/12 macaques (Fig. 1r, Supplementary Table 2 and Extended Data Fig. 2c) and 12/12 Baboons (Fig. 1s and Extended Data Fig. 3d-g), generally composed of macrophages and lymphocytes that expanded the alveolar septa (Fig. 1r,s and Extended Data Fig. 2d-g), with variable neutrophil infiltrates (Extended Data Figs. 2e and 3d,e,h,i) or fibrosis (Fig. 1r,s and Extended Data Figs. 2f,g and $3 \mathrm{~h}, \mathrm{i})$. A subset of six marmosets was euthanized at 3 d.p.i. $(n=2)$, while others were necropsied at 14 d.p.i. Interstitial and alveolar pneumonitis was observed in the marmosets (Fig. 1t), although not as prevalent as in macaques or baboons. Thus, our results show that three NHP genera develop different degrees of COVID-19 when evaluated side by side, with baboons exhibiting moderate to severe pathology, macaques exhibiting moderate pathology and old marmosets exhibiting mild pathology. The pulmonary inflammation score was significantly different between macaques and baboons,

Fig. 1 | SARS-CoV-2 RNA and histopathology in rhesus macaques, baboons and marmosets. a-d, vRNA in BAL fluid (a), NS (b) and RS (c) collected longitudinally, and lung tissue homogenates (d) collected at the end point (14-17 d.p.i.) from rhesus macaques infected with SARS-CoV-2. e-h, vRNA in BAL fluid (e), NS (f) and RS (g) collected longitudinally, and lung tissue homogenates (h) at the end point (14-17 d.p.i.) from baboons infected with SARS-CoV-2. $n=12$. i-I, Comparison of vRNA in BAL fluid (i), NS (j), RS ( $(\mathbf{k})$ and lungs (I) of rhesus macaques and baboons infected with SARS-CoV-2. $\mathbf{m}, \mathbf{n}$, To estimate the persistence of replicative virus, we performed subgenomic RNA estimation on end-point lung samples of rhesus macaques ( $\mathbf{m}$ ) and baboons $(\mathbf{n}) . n=12$. o,p, vRNA in nasal wash (o) and oral (p) longitudinal swabs. $n=6$ (0-3 d.p.i.) and $n=4$ (6-14 d.p.i.). q, vRNA was also measured in lung homogenates of marmosets at the end point. $n=2$ (3 d.p.i.) and $n=4$ (14 d.p.i.). Statistical analysis was performed using one-way (a-c and $\mathbf{e}-\mathbf{g}$ ) and two-way (i-k) repeated-measures analysis of variance (ANOVA) with Geisser-Greenhouse correction for sphericity and Tukey post hoc correction for multiple testing (GraphPad Prism 8). r, Histopathological analysis in infected rhesus macaques revealed regionally extensive interstitial lymphocytes, plasma cells, lesser macrophages and eosinophils expanding the alveolar septa (bracket) and alveolar spaces filled with macrophages (asterisks). The normal alveolar wall is indicated (arrow) for comparison (top). Bottom, alveolar spaces with extensive interstitial alveolar wall thickening by deposits of collagen (asterisks) and scattered alveolar macrophages (arrow). s, Histopathological analysis in infected baboons also revealed regionally extensive interstitial lymphocytes, plasma cells, lesser macrophages and eosinophils expanding the alveolar septa (bracket) and alveolar spaces filled with macrophages (asterisk) (top). Bottom, alveolar wall thickening by interstitial deposits of collagen (asterisk), alveoli lined by occasional type-II pneumocytes (arrowheads) and alveolar spaces containing syncytial cells (arrows) and alveolar macrophages. $\mathbf{t}$, Histopathological analysis in marmosets revealed a milder form of interstitial lymphocytes, and macrophages recruited to the alveolar space. Scale bars, $100 \mu \mathrm{m}(\mathbf{r}, \mathbf{s}$ (top), $\mathbf{t}$ (top)) and $50 \mu \mathrm{m}$ ( $\mathbf{s}$ (bottom) and $\mathbf{t}$ (bottom)). u,v, Comparison of the lung inflammation score (u) and end-point viral titre (v) of infected rhesus macaques and baboons. $n=12$. Statistical analysis was performed using one-tailed Mann-Whitney $U$-tests ( $\mathbf{m}, \mathbf{n}, \mathbf{u}$ and $\mathbf{v}$ ). For $\mathbf{a}-\mathbf{q}$, $\mathbf{u}$ and $\mathbf{v}$, the shapes indicate old (triangles) and young (diamonds) rhesus macaques; old (inverted triangles) and young (squares) baboons; and old marmosets (hexagons). The different colours represent individual animals (Supplementary Table 1). For $\mathbf{a}, \mathbf{b}, \mathbf{e}-\mathbf{g}, \mathbf{i}-\mathbf{k}, \mathbf{m}-\mathbf{o}, \mathbf{u}$ and $\mathbf{v}, P$ values are indicated above the plots. Data are mean (i, $\mathbf{k}$ and $\mathbf{q}$ ) or mean \pm s.e.m. (a-h, $\mathbf{m}-\mathbf{o}, \mathbf{u}$ and $\mathbf{v})$. 
with greater inflammation in baboon lungs (Fig. 1u); however the vRNA levels did not differ statistically (Fig. 1v).

Longitudinal SARS-CoV-2 infection in rhesus macaques, baboons and marmosets demonstrates heterogeneity in radiological and clinical outcomes across age and species. All SARS-CoV2-infected macaques (Fig. 2a and Supplementary Table 4) and baboons (Fig. 2b and Supplementary Table 5) exhibited low baseline chest X-ray (CXR) scores (Fig. 2a,b and Supplementary Tables 4 and 5) with no age differences (Fig. 2a,b), but there were significantly

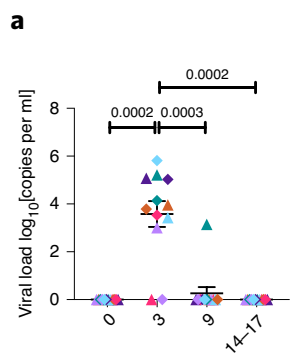

Time (d.p.i.)

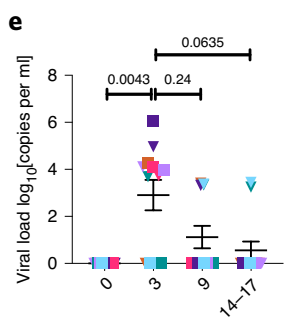

Time (d.p.i.)

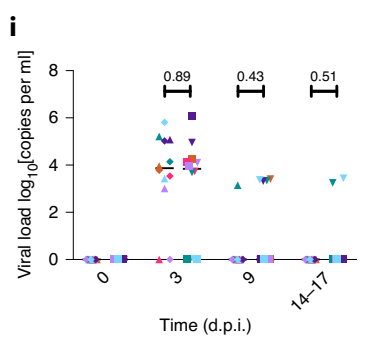

m

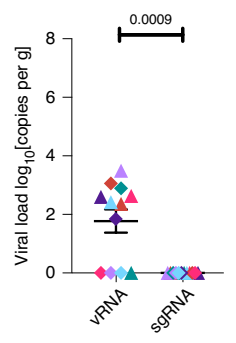

n

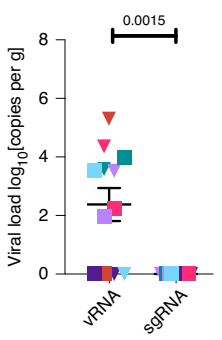

o

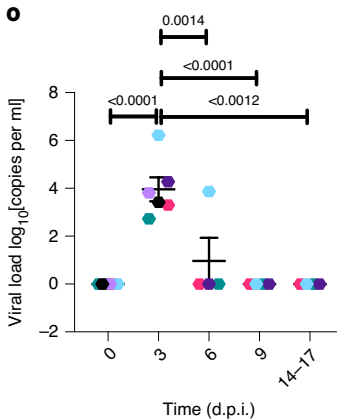

$\mathbf{p}$
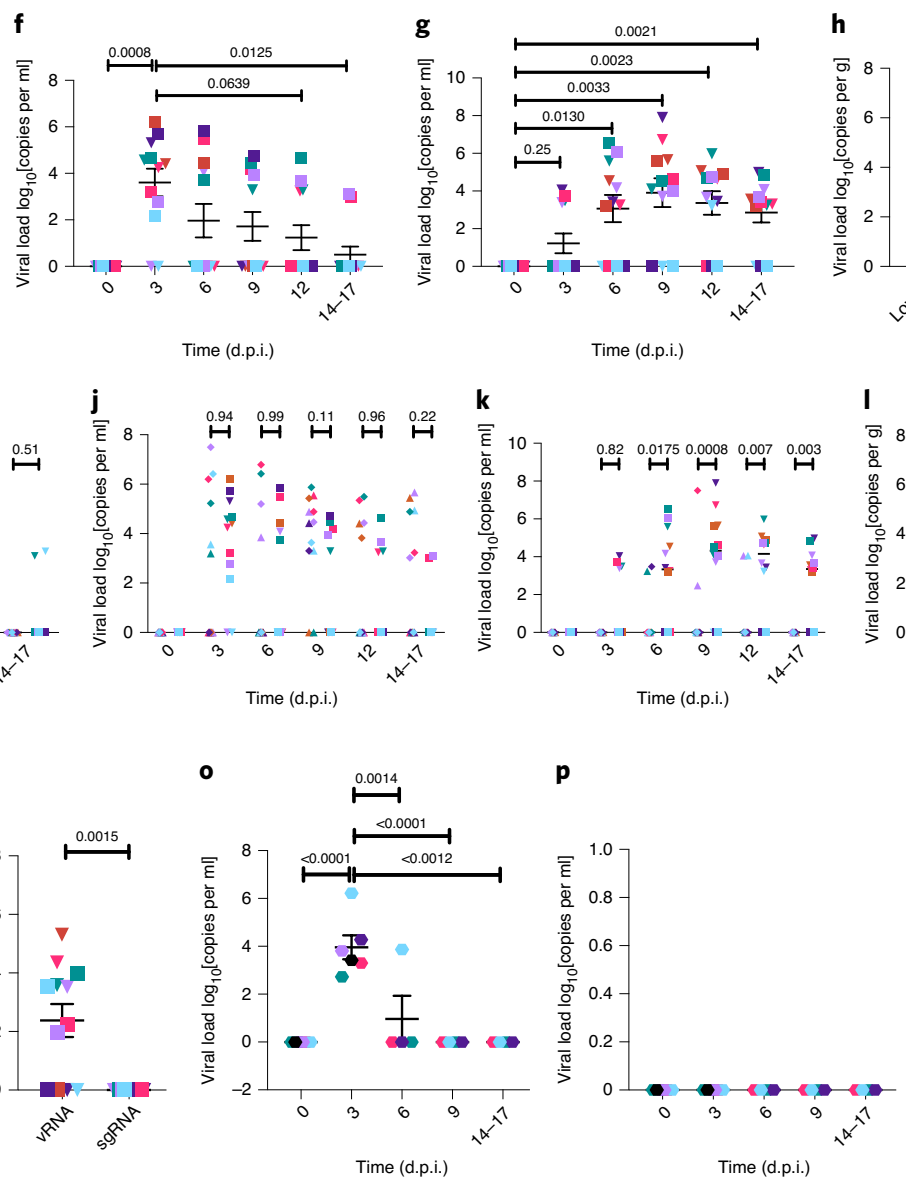

Time (d.p.i.)
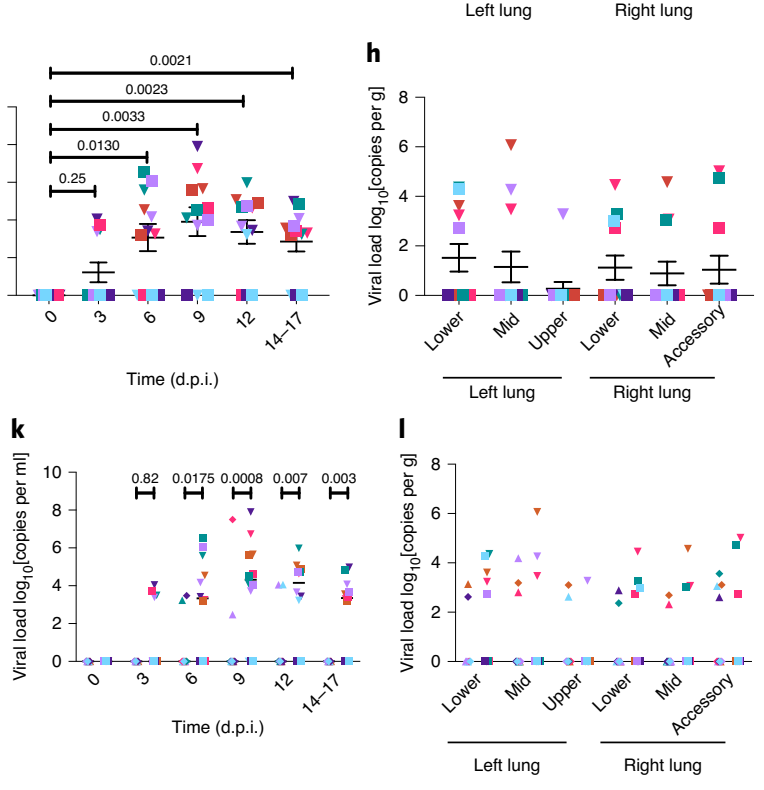

$\mathbf{q}$
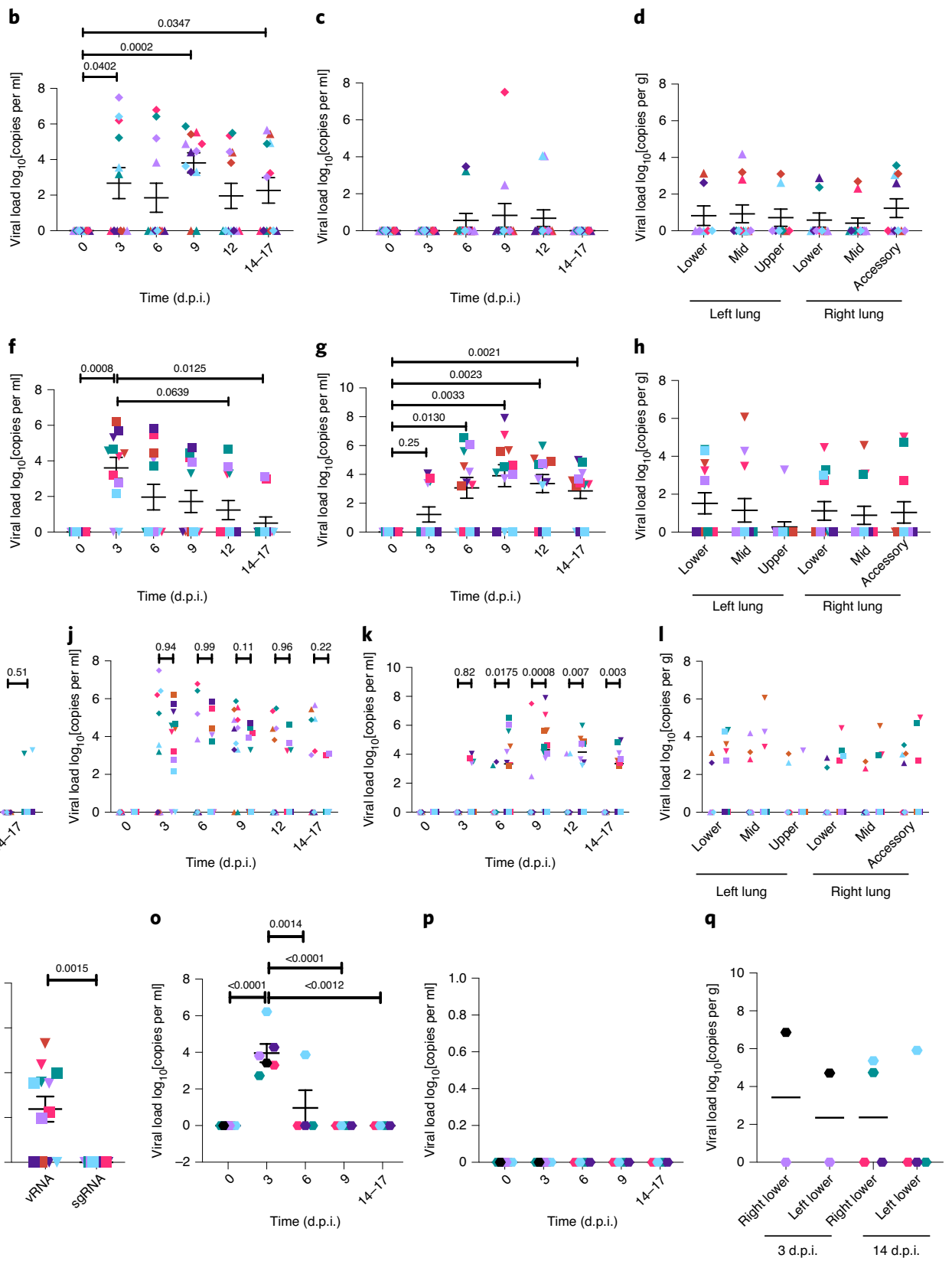
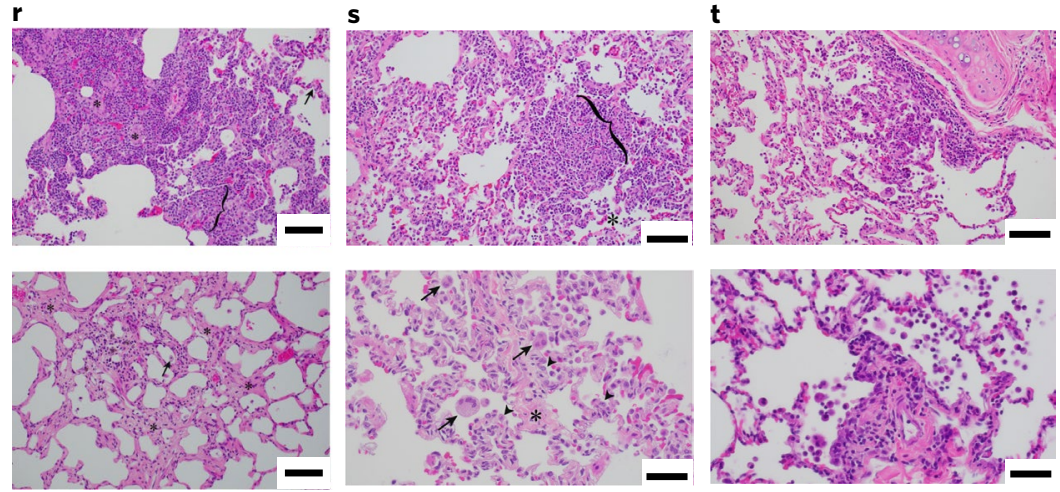

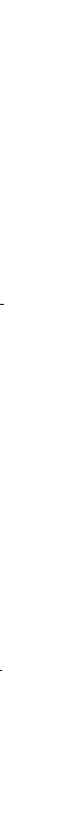

$\sqrt{2}$
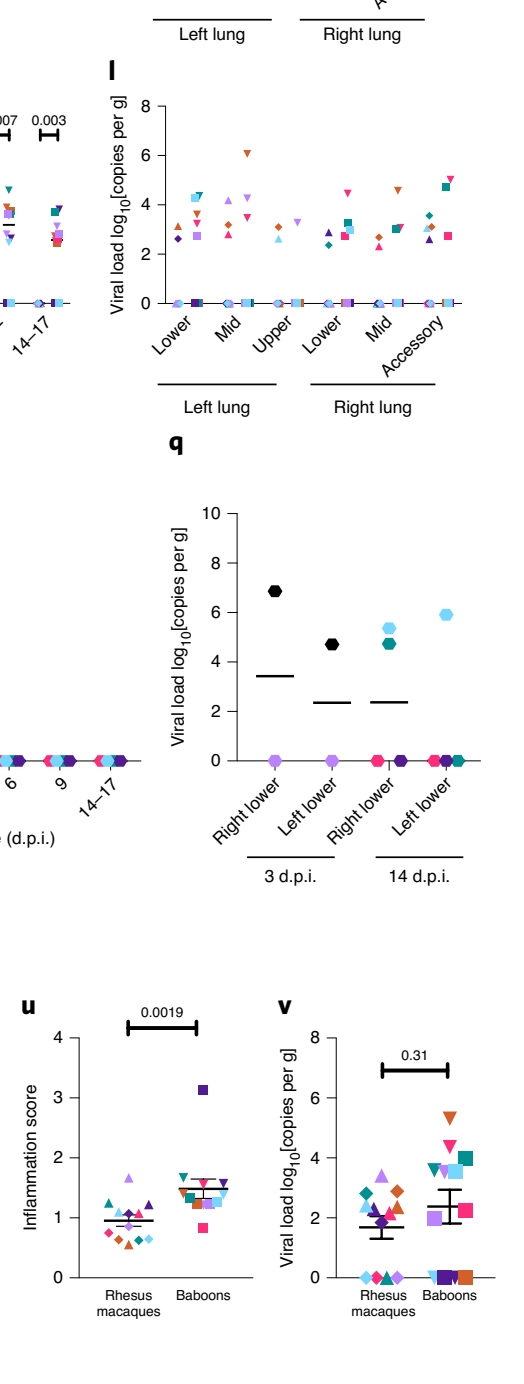
higher CXR scores in baboons compared with in macaques (Fig. 2c) at day 9 and the study end. Several infected macaques showed changes that were consistent with pneumonia (Supplementary Table 4) with peak severity at 3-6d.p.i. and a decline by the study end (Fig. 2a and Supplementary Table 4). Examples of extensive pneumonia were seen in CXRs in macaques at 6 d.p.i. with subsequent resolution at the end point (Supplementary Fig. 1a-c). Several animals exhibited multilobe alveolar infiltrates and/or interstitial opacities at 6 d.p.i., while others exhibited progressive, moderate to severe interstitial and alveolar infiltrates at 6 d.p.i. that resolved by day 14 . By contrast, CXRs of all procedure control animals (which underwent repeated BAL procedures) revealed minimal to no findings.

High-resolution lung CT imaging was performed before and after SARS-CoV-2 infection in six young and six old macaques (Fig. 2d-m). CT scans for baboons and both CT scans and CXR imaging for marmosets were not feasible. Pneumonia was present in all animals after infection, but to a significantly higher degree in old macaques compared with in young macaques (Extended Data Fig. 4 and Supplementary Table 6). At 6 d.p.i., severe patchy alveolar patterns were observed in some lobes, while others had milder interstitial patterns, with moderate to severe ground-glass opacities primarily in old macaques (Extended Data Fig. 4a-f). Resolution of many ground-glass opacities and nodular as well as multifocal lesions was observed in all animals at 12 d.p.i. (Fig. $2 \mathrm{~d}$ and Extended Data Fig. 4a,b,d-f). At 12 d.p.i., all but one of the old macaques exhibited a normal or nearly normal CT scan. Findings in one old macaque were considerably improved but retained patchy ground-glass opacities in all lobes and alveolar patterns in some lobes at 12 d.p.i. (Extended Data Fig. 4c). This animal had the highest overall score on the basis of CT scans (Fig. 2d) and CXRs (Fig. 2a,b). These results suggest that multilobe pneumonia may persist for longer in some old macaques. Hyperdensity analysis revealed a significant progressive increase in involved lung volume at 6 d.p.i., which normalized by 12 d.p.i. (Fig. $2 \mathrm{e}-\mathrm{m}$ ).

Early events in SARS-CoV-2 infection of rhesus macaques. We performed an independent acute $3 \mathrm{~d}$ infection study of rhesus macaques that included extensive imaging and immunological readouts. Four rhesus macaques (Supplementary Table 1) were infected as described above and all of the animals developed clinical signs of viral infection with a doubling of serum C-reactive protein (CRP) levels relative to the baseline (Fig. 2n); significantly decreased serum albumin (Fig. 2o) and haemoglobin (Fig. 2p) levels, indicating viral-induced anaemia; and progressively increasing total serum $\mathrm{CO}_{2}$ levels (Extended Data Fig. 5a), indicative of pulmonary dysfunction.

As before, vRNA was detected in BAL, NS and BS at 1-3 d.p.i. (Fig. 2q-s) and in RS at 1 d.p.i. (Extended Data Fig. 5i). At necropsy (3 d.p.i.), SARS-CoV-2 vRNA was detected in 23/24 random lung sections analysed. We detected 6-8 log-transformed copies per $100 \mathrm{mg}$ of lung tissue from every lobe (Fig. 2t). The $\sim 4 \log$ increase in BAL vRNA from 1 to 2 d.p.i. (Fig. 2q) was consistent with early active replication of SARS-CoV-2, a finding that was verified by significant subgenomic vRNA levels $(6 \log$ ) at 3 d.p.i. (Fig. $2 \mathrm{u}$ ), which was $\sim 2 \log$ lower than detected vRNA (Fig. $2 v$ ). Thus, SARS-CoV-2 induces a rapid replicative response in the lungs that is evident within $3 \mathrm{~d}$ and that subsides by two weeks, with low vRNA (Fig. $2 \mathrm{w}$ ) and no subgenomic vRNA detected in lung samples at 14-17 d.p.i. (Fig. 2x). The vRNA titres in BAL and NS of rhesus macaques demonstrated a strong positive correlation with the vRNA titres in the lungs at both end points (Extended Data Fig. 1f-h).

Examination at day 3 necropsy revealed findings of interstitial and alveolar pneumonia (Fig. 2y). A summary of the histopathological findings is provided in Supplementary Table 7. The lungs were again the most affected organ (Fig. 2y, Supplementary Table 7 and Extended Data Fig. 6). Multifocal, mild to moderate interstitial pneumonia characterized by infiltrates of neutrophils, macrophages, lymphocytes and eosinophils was present in all four animals (Fig. 2y (bottom) and Extended Data Fig. 6d,e,g,h), and was accompanied by variable fibrosis (4/4; Extended Data Fig. 6e), fibrin deposition (3/4; Extended Data Fig. 6c) and vasculitis (3/4; Extended Data Fig. 6f). All four macaques exhibited the following: (1) syncytial cells in the epithelial lining and/or alveolar lumen (Extended Data Fig. 6e,g,k); (2) bronchitis characterized by infiltrates of eosinophils within the bronchial wall and epithelium (Fig. 2y (top) and Extended Data Fig. 6i-k); (3) bronchus-associated lymphoid tissue hyperplasia (Extended Data Fig. 6i); and (4) minimal to moderate lymphoplasmacytic and eosinophilic tracheitis and rhinitis.

Fluorescence immunohistochemical analysis revealed SARS-CoV-2 proteins in the lungs (Fig. $2 \mathrm{z}$ (top) and Supplementary Figs. 2a,g and 3a,d,g,j), nasal epithelium (Fig. 2z (bottom) and Supplementary Figs. $2 \mathrm{~b}, \mathrm{~h}$ and $3 \mathrm{~b}, \mathrm{e}, \mathrm{h}, \mathrm{k}$ ) and tonsils (Supplementary Figs. 2c,i and 3c,f,i,l). In all tissues, including the lungs (Fig. 2y (top) and Supplementary Fig. 2a,g), nasal epithelium (Fig. 2z (top) and Supplementary Fig. 2b,h) and tonsils (Supplementary Fig. 2c,i), N antigen was detected in cells expressing ACE2 and in adjacent cells, consistent with SARS-CoV-2 using ACE2 to bind to, internalize and proliferate inside cells ${ }^{7,8}$. ACE2 protein levels were much lower in

Fig. 2 | Radiology and correlates of SARS-CoV-2 infection in rhesus macaques and baboons. a-c, CXR scores in macaques (a; $n=12)$ and baboons (b; $n=12)$, and comparative CXR scores of infected rhesus macaques and baboons (c). d, CT scores in macaques. $n=6$. e,i, Three-dimensional (3D) reconstruction of the region of interest (ROI) volume representing the location of the lesion at day $6(\mathbf{e})$ and day 12 (i). $\mathbf{f}-\mathbf{h}, \mathbf{j}-\mathbf{I}$, Representative longitudinal $(\mathbf{f}, \mathbf{j})$, vertical $(\mathbf{g}, \mathbf{k})$ and transverse $(\mathbf{h}, \mathbf{I})$ images for the quantification of lung lesions at day $6(\mathbf{f}-\mathbf{h})$ and day $12(\mathbf{j}-\mathbf{I})$; the teal area represents normal-intensity voxels and the yellow areas represent hyperdense voxels. $\mathbf{m}$, The percentage change in lung hyperdensity in animals infected with SARS-CoV-2 over 6 d.p.i. compared with over 12 d.p.i. $n=6$. $\mathbf{n}-\mathbf{s}$, Clinical correlates of early SARS-CoV-2 infection in rhesus macaques (circles) over 0-3 d.p.i. showing changes in serum CRP (n), albumin (ALB) (o), haemoglobin (HGB) content (p) in peripheral blood; vRNA in BAL fluid (q) and NS (r) and BS (s) collected longitudinally. $\mathbf{t}, \mathbf{u}, \mathrm{vRNA}(\mathbf{t})$ and subgenomic RNA (u) was measured in lung tissue homogenates at the end point (3 d.p.i.). v, Side-by-side comparison of vRNA and subgenomic RNA. For $\mathbf{n}-\mathbf{v}$, the different colours represent individual animals (Supplementary Table 1). For $\mathbf{n}-\mathbf{v}, n=4$. w, $\mathbf{x}$, Comparison of the viral titre (w) and lung subgenomic RNA ( $\mathbf{x}$ ) of infected rhesus macaques between a short ( $3 \mathrm{~d}$ ) and a long (14-17d) study. $n=4$ ( $3 \mathrm{~d}$ ) and $n$ $=12(14-17 \mathrm{~d})$. Statistical analysis was performed using Mann-Whitney U-tests. $\mathbf{y}$, Haematoxylin and eosin staining was performed on formalin-fixed paraffin-embedded lung sections from infected animals for pathological analysis. Top, histopathological analysis revealed bronchitis characterized by infiltrates of macrophages, lymphocytes, neutrophils and eosinophils that expanded the wall (bracket) and, along with syncytial cells (arrows), filled the bronchiole lumen and the adjacent alveolar spaces. Bottom, suppurative interstitial pneumonia with type-Il pneumocyte hyperplasia (arrowheads); the alveolar space is filled with neutrophils, macrophages and fibrin (asterisk). The bracket denotes the alveolar space. Scale bars, $100 \mu \mathrm{m}$ (top) and $50 \mu \mathrm{m}$ (bottom). $\mathbf{z}$, Multilabel confocal immunofluorescence microscopy of the lungs (top) and nasal epithelium (bottom) at $\times 63$ magnification with nucleocapsid (N)-specific antibodies (turquoise) 4,6-diamidino-2-phenylindole (DAPI) (blue) and ACE2 (magenta). Scale bars, $20 \mu \mathrm{m}$. Undetectable viral titres were represented as one copy. Statistical analysis was performed using one-way (a, $\mathbf{b}$ and $\mathbf{n}$-s) and two-way (c) repeated-measures ANOVA with Geisser-Greenhouse correction for sphericity and Tukey post hoc correction for multiple testing (GraphPad Prism 8); and with one-tailed Mann-Whitney $U$-tests $(\mathbf{d}, \mathbf{m}$ and $\mathbf{v}-\mathbf{x})$. For $\mathbf{a}-\mathbf{d}, \mathbf{m}-\mathbf{s}$ and $\mathbf{v}-\mathbf{x}, P$ values are indicated above the plots. Data are mean $(\mathbf{t}-\mathbf{v})$ or mean \pm s.e.m. $(\mathbf{a}, \mathbf{b}, \mathbf{d}, \mathbf{m}-\mathbf{s}, \mathbf{w}$ and $\mathbf{x})$. 
lung tissues derived from naive animals compared with SARS-CoV2 -infected animals (Supplementary Fig. 2m,n), a finding that is consistent with a recent RNA-sequencing analysis showing that ACE2 is upregulated after infection, especially in young macaques ${ }^{9}$. The majority of S signal was detected in the epithelial layer with discrete distribution throughout the lung tissue (Supplementary Fig. 3a,d).

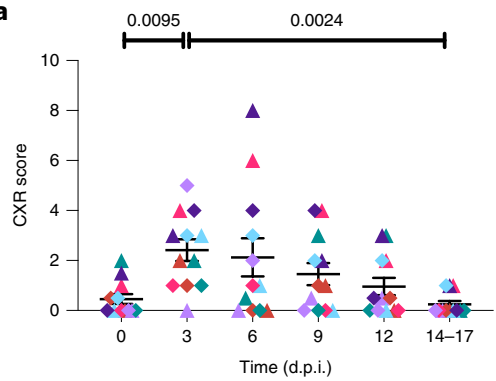

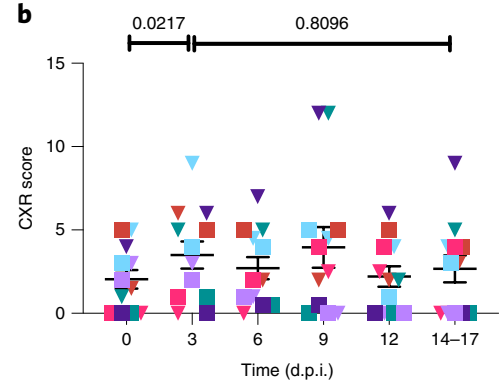

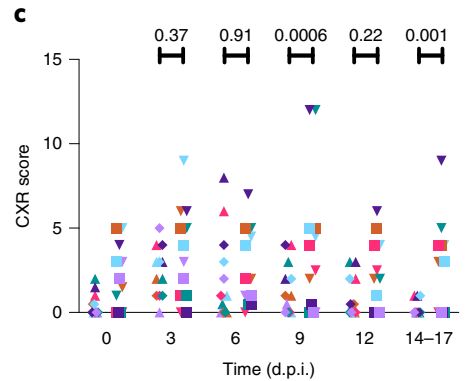

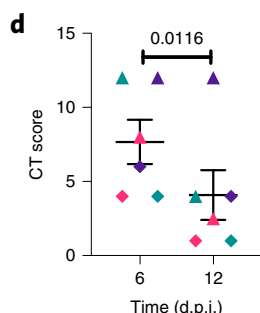

Day 6

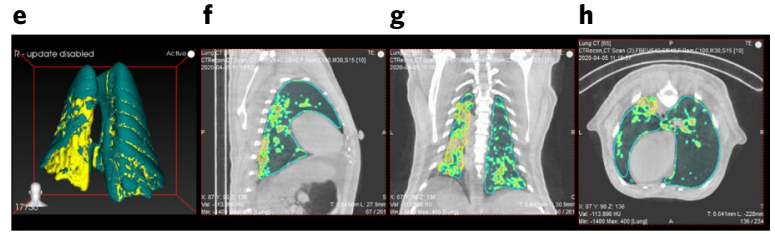

Day 12

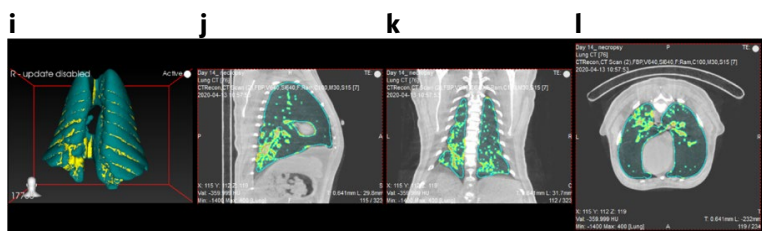

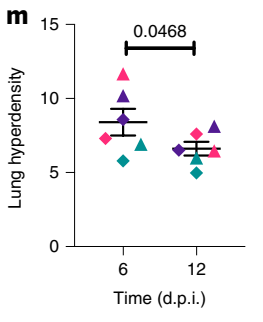
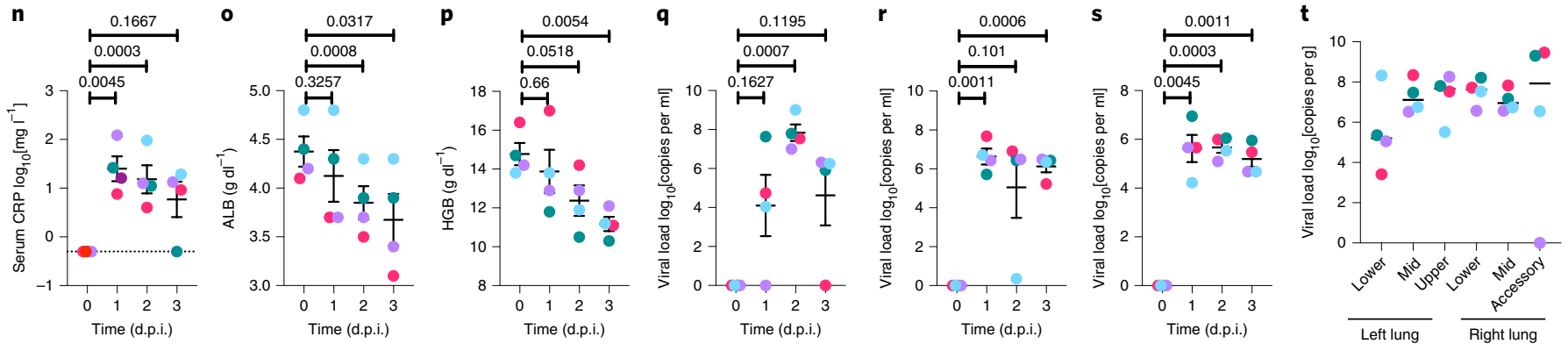
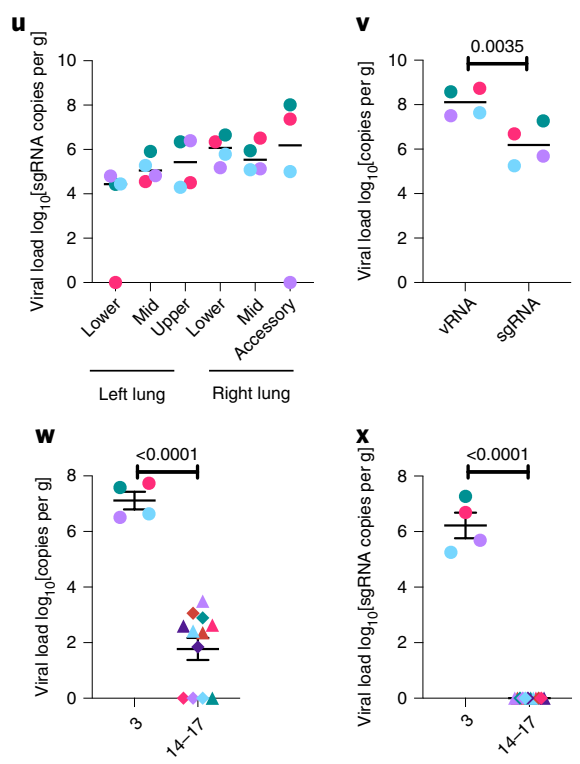

Time (d.p.i.)

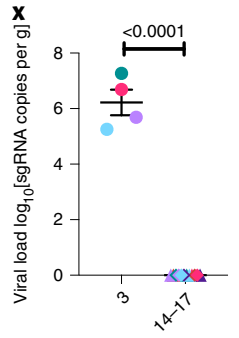

Time (d.p.i.)
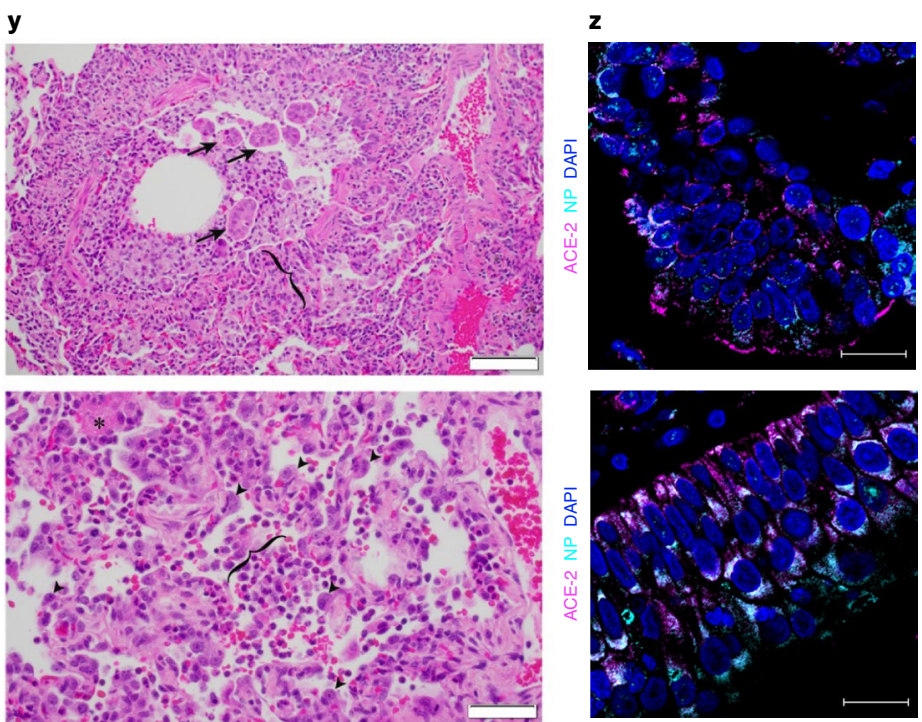

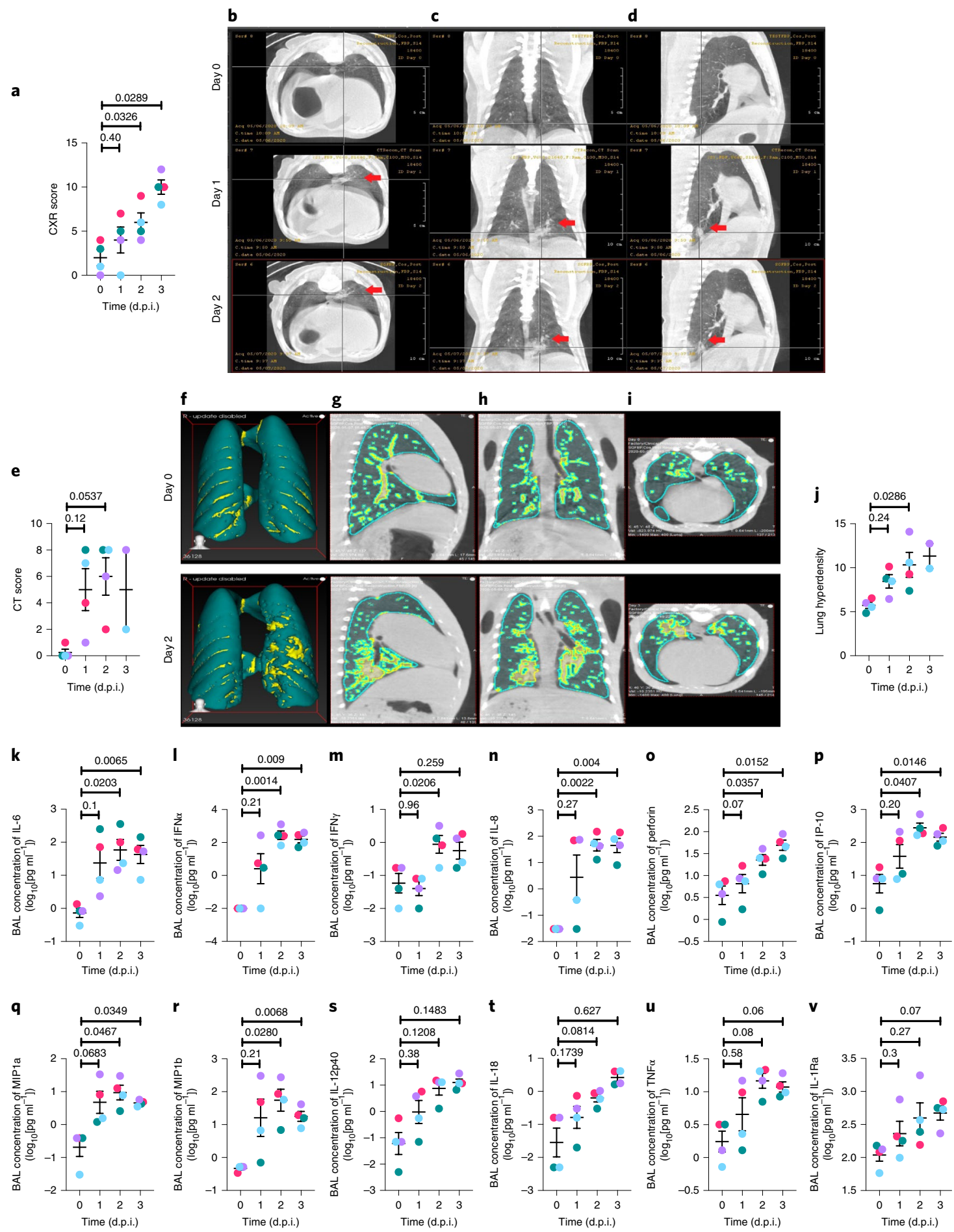

Fig. 3 | The alveolar compartment over 0-3 d.p.i. in infected rhesus macaques. a,e, CXR (a) and CT (e) scores of rhesus macaques (circles) over 0-3 d.p.i. The different colours represent individual animals (Supplementary Table 1). b-d, Representative CT scan images performed on day 0-2 d.p.i. show transverse (b), vertical (c) and longitudinal (d) views of the left caudal lobe ground-glass opacity at 1 d.p.i. (middle), 2 d.p.i. (bottom) and the baseline at 0 d.p.i. (top). The CT scans in $\mathbf{b}-\mathbf{d}$ revealed evidence of pneumonia and lung abnormalities in the infected animals relative to controls, which resolved between 1 and 2 d.p.i. (red arrows). $\mathbf{f}, 3 \mathrm{D}$ reconstruction of the ROI volume representing the location of the lesion. $\mathbf{g}$-i, Representative longitudinal $(\mathbf{g})$, vertical (h) and transverse (i) images for the quantification of lung lesions; the teal area represents normal-intensity lung voxels, while the yellow areas represent hyperdense voxels. $\mathbf{j}$, The percentage change in lung hyperdensity in SARS-CoV-2-infected animals over days 1-3 d.p.i. compared with the baseline. k-v, Simultaneous analysis of multiple cytokines using Luminex technology in BAL fluid of rhesus macaques over 0-3 d.p.i. revealed that SARS-CoV-2 induced alveolar inflammation, showing increased levels of IL-6 (k), IFN $\alpha(\mathbf{I})$, IFN $\gamma(\mathbf{m})$, IL-8 (n), perforin (o), IP-10 (p), MIP1a (q), MIP1b $(\mathbf{r})$, IL-12p40 (s), IL-18 (t), TNF $\alpha(\mathbf{u})$ and IL-1Ra (v), expressed as $\log _{10}$-transformed concentration in pg ml ${ }^{-1}$ of BAL fluid. $n=4$. For $\mathbf{a}$ and $\mathbf{k}-\mathbf{v}$, statistical analysis was performed using one-way repeated-measures ANOVA with Geisser-Greenhouse correction for sphericity and Tukey post hoc correction for multiple testing (GraphPad Prism 8). For e and $\mathbf{j}$, statistical analysis was performed using ordinary one-way ANOVA with Dunnett's post hoc test. $n=4$ (0-2 d.p.i.) and $n=2$ (3 d.p.i.). For $\mathbf{a}, \mathbf{e}$ and $\mathbf{j}-\mathbf{v}, P$ values are indicated above the plots; data are mean \pm s.e.m. 
In the nasal cavity, virus was observed in epithelial lining cells (Supplementary Fig. 3b,h), but was distributed throughout tonsillar tissue (Supplementary Fig. 2c,i). Thus, viral replication is supported in the upper and lower lung compartments during the first $3 \mathrm{~d}$ of infection and viral antigens are detected at high levels in the lungs.

CXRs of all four infected macaques showed progressive increase in CXR abnormality scores (Fig. 3a and Extended Data Fig. 7a). The CXR scores at 2 and 3 d.p.i. were significantly increased relative to the baseline (Fig. 3a), with partial resolution of specific lesions at 2 or 3 d.p.i. (Extended Data Fig. 7a). There were mild to severe multifocal interstitial-to-alveolar patterns with soft tissue opacities in various lobes or diffusely in some animals, with more-severe abnormalities in the lower lung lobes, and with the most severe findings at 3 d.p.i. (Extended Data Fig. 7a). Lung CT scans showed increased multifocal pulmonary infiltrates with ground-glass opacities in various lobes, linear opacities in the lung parenchyma, nodular opacities in some lung lobes and increased soft tissue attenuation extending primarily adjacent to the vasculature within 1 d.p.i. (Fig. $3 \mathrm{~b}-\mathrm{d}$ and Extended Data Fig. 7b-e). In some of the animals, multifocal alveolar pulmonary patterns and interstitial opacities were observed in lobe subsections, with soft tissue attenuation and focal border effacement with the pulmonary vasculature. Features intensified at 2-3d.p.i., primarily in the lung periphery, but also adjacent to the primary bronchus and the vasculature (Fig. 3b-d and Extended Data Fig. 7b). In other animals, progressive alveolar or interstitial pulmonary patterns were observed at 2 d.p.i. (Fig. $3 b-d$ ). Although ground-glass opacities intensified at 2 d.p.i. in some lobes, others resolved (Extended Data Fig. 7c,d). In one animal, the individual nodular pattern at 1 d.p.i. evolved to a multifocal soft tissue nodular pattern in multiple lobes with associated diffuse ground-glass opacities (Extended Data Fig. 7d). At 3 d.p.i., persistent, patchy, fairly diffuse ground-glass pulmonary opacities existed in many lung lobes with multifocal nodular tendency (Extended Data Fig. 7e). Overall, CT abnormality scores continuously increased over 3 d.p.i. (Fig. 3e). The percentage change in the hyperdensity volume was calculated using CT scans to quantify pathological changes over time ${ }^{10}$. We observed a substantial increase in lung hyperdense areas from 1 to 3 d.p.i. (Fig. $3 \mathrm{f}-\mathrm{j}$ ). Together, CXR and CT scans revealed moderate multilobe pneumonia in all of the infected animals (Fig. 3j), confirming the histopathology results (Fig. $2 y$ and Extended Data Fig. 6) in the very early phase of SARS-CoV-2 infection in macaques.

IL-6, IFN $\alpha$, IFN $\gamma$, IL-8, perforin, IP-10, MIP1 $\alpha$ and MIP1 $\beta$ (Fig. $3 \mathrm{k}-\mathrm{v}$ ) were all significantly elevated in BAL fluid of acutely infected macaques. Of particular interest was the elevated IFN $\alpha$ (Fig. 31), which has critical anti-viral activity, including against SARS-CoV-2 (ref. ${ }^{11}$ ). Expression of a downstream type-I-IFN-regulated gene CXCL10 (which encodes IP-10), which promotes the recruitment of $\mathrm{CXCR}^{+} \mathrm{T}$ helper $1\left(\mathrm{~T}_{\mathrm{H}} 1\right)$ cells, was also increased (Fig. 3p). Macaques therefore mount an early anti-viral response to SARS-CoV-2 infection. Type-I IFNs and IL-6 are key components of a 'cytokine storm' that promote acute respiratory-distress syndrome, which is associated with both SARS-CoV-1 and SARS-CoV-2 when induced uncontrollably ${ }^{12}$. Of the plasma cytokine levels tested (Extended Data Fig. 8), IFN $\alpha$ and IP-10 were also significantly elevated at 2 and 3 d.p.i. (Extended Data Fig. 8b,f). Thus, clinical, imaging, pathology and cytokine analyses provided evidence for an acute SARS-CoV-2 infection in macaques that leads to moderate pneumonia with early activation of anti-viral responses.

Myeloid cell response in the lungs of infected rhesus macaques. BAL predominantly comprises alveolar macrophages (AMs) in healthy lungs ${ }^{13}$. BAL and peripheral blood cellular composition $^{14,15}$ of SARS-CoV-2-infected macaques in our longitudinal study showed substantially altered immune cell composition and responses at necropsy. Infection moderately increased the proportions of BAL myeloid cells, interstitial macrophages (IMs; Fig. 4a,e), neutrophils (Fig. 4c,g) and plasmacytoid dendritic cells (pDCs; Fig. 4d,h)) at 3 d.p.i. with no age effect. By contrast, resident AM levels decreased significantly at 3 d.p.i. (Fig. 4b,f). The increase in myeloid subpopulations at 3 d.p.i. was highly correlated with vRNA levels (Fig. 4i,j). Multilabel confocal imaging of Ki67 staining showed that few of the virally infected cells in lung tissue actively proliferated (Fig. $4 \mathrm{k}-\mathrm{m}$ ). Neutrophils (Fig. $4 \mathrm{k}-\mathrm{m}$ and Extended Data Fig. 9a,i), macrophages (Fig. $4 \mathrm{n}-\mathrm{p}$ and Extended Data Fig. 9b,j) and pDCs (Fig. 4q-s and Extended Data Fig. 8c,k) recruited to the lung harboured high levels of viral proteins (Fig. 4k-s and Extended Data Fig. 9). Furthermore, resident epithelial cells, type-1 and type-2 pneumocytes, all harboured viral antigens (Fig. 4t-v and Extended Data Fig. 9d,l). These are also reported to be the primary alveolar cells with high ACE2 expression and SARS-CoV-2 signal in $\mathrm{NHPs}^{16}$ and humans ${ }^{17}$. These data suggest that early infection of resident alveolar cells might trigger the cytokine storm that induces rapid lung influx of specialized myeloid cell subsets that are known to express type-I IFNs and other proinflammatory cytokines, and is a key event in the control of SARS-CoV-2 infection. Relative to AMs, IMs have a shorter half-life, exhibit continuous turnover, help to maintain homeostasis and protect against continuous pathogen exposure from the environment ${ }^{18}$. Increased lung recruitment of pDCs suggests that they are a potentially important feature of protection against advanced COVID-19 because they are a major source of type-I IFNs, such as IFN $\alpha$, which is elevated in BAL within 1-3 d.p.i. (Fig. 31 and Extended Data Fig. 8b).

T-cell, antibody and cytokine responses in the lungs of infected rhesus macaques. Macaque infection resulted in a significant T-cell influx into the alveolar space by 3 d.p.i., which normalized by 9 d.p.i. (Fig. 5a-c) and correlated significantly with BAL viral titre (Fig. 5d-f). After infection, $\mathrm{CD}^{+} \mathrm{T}$ cells in BAL expressed significantly lower levels of antigen experience/tissue residence (CD69), $\mathrm{T}_{\mathrm{H}} 1$ (CXCR3), memory (CCR7) and activation (HLA-DR; Fig. 5g-k) markers. By contrast, the level of $\mathrm{CD}^{+}{ }^{+} \mathrm{T}$ cells expressing PD-1 and LAG-3 (Fig. 5l,m) was significantly increased. CXCR3 expression on $\mathrm{CD}^{+}$ $\mathrm{T}$ cells showed significant negative correlation with viral titre,

Fig. 4 | Accumulation of myeloid cells in BAL of infected rhesus macaques. a-h, Flow cytometry analysis of BAL IMs (a,e), AMs (b,f), neutrophils $(\mathbf{c}, \mathbf{g})$ and pDCs $(\mathbf{d}, \mathbf{h})$. For $\mathbf{a}-\mathbf{d}$, data are combined for age $(n=12)$. For $\mathbf{e}-\mathbf{h}$, data are split by age $(n=6)$. Statistical analysis was performed using one-way (a-d) and two-way (e-h) repeated-measures ANOVA with Geisser-Greenhouse correction for sphericity and Tukey post hoc correction for multiple testing (GraphPad Prism 8). The shapes indicate old (triangles) and young (diamonds) animals. The different colours represent individual animals (Supplementary Table 1). $n=12$. i,j, Spearman's rank correlation between the cellular fraction and $\log _{10}$-transformed vRNA copy number in BAL was calculated (i) and the corresponding values for the Spearman's rank correlation coefficient ( $\mathbf{j}$, left) and $P$ values ( $\mathbf{j}$, right) are shown. Multilabel confocal immunofluorescence microscopy of formalin-fixed paraffin-embedded lung sections from rhesus macaques infected with SARS-CoV-2 with a high viral titre at 3 d.p.i., stained with DAPI (blue) (k-v); SARS-CoV-2 spike-specific antibodies (k-s) (turquoise); Ki67 (magenta) and neutrophil marker CD66abce (yellow) (k-m); pan-macrophage marker CD68 (magenta) (n-p); HLA-DR (magenta) and pDC marker CD123 (yellow) (q-s); SARS-CoV-2 nucleocapsid-protein-specific antibodies (turquoise), pan-cytokeratin (magenta) and thyroid transcription factor-1 (yellow) (t-v). For k-v, scale bars, $20 \mu \mathrm{m}$ ( $\times 63$ magnification; $\mathbf{k}, \mathbf{n}, \mathbf{q}$ and $\mathbf{t}$ ), $50 \mu \mathrm{m}$ ( $\times 20$ magnification; $\mathbf{l}, \mathbf{o}, \mathbf{r}$ and $\mathbf{u})$ and $100 \mu \mathrm{m}(\times 10$ magnification; $\mathbf{m}, \mathbf{p}, \mathbf{s}$ and $\mathbf{v})$. For $\mathbf{a}-\mathbf{h}, P$ values are indicated above the plots; data are mean \pm s.e.m. 
whereas PD1 expression was positively, albeit not significantly, correlated (Fig. 5n-p). A similar effect was observed in BAL CD8 ${ }^{+}$T-cell subsets, in which CD69, CXCR3 and CCR7 expression (Fig. 5q-u) was significantly reduced after infection, whereas PD-1 and LAG-3 expression (Fig. 5v,w) was significantly increased. PD1 expression on CD8 subsets was positively correlated with BAL viral titre,
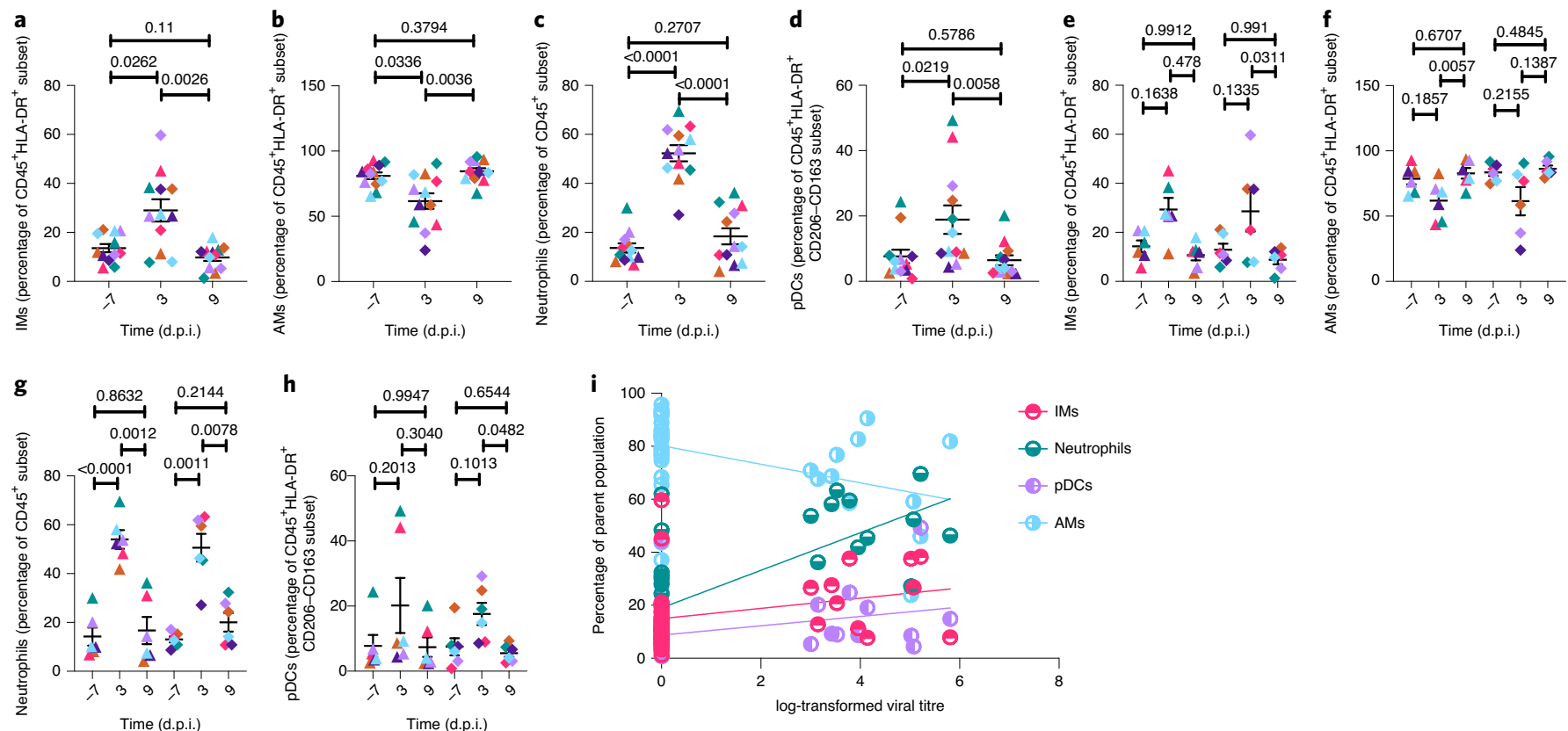

j
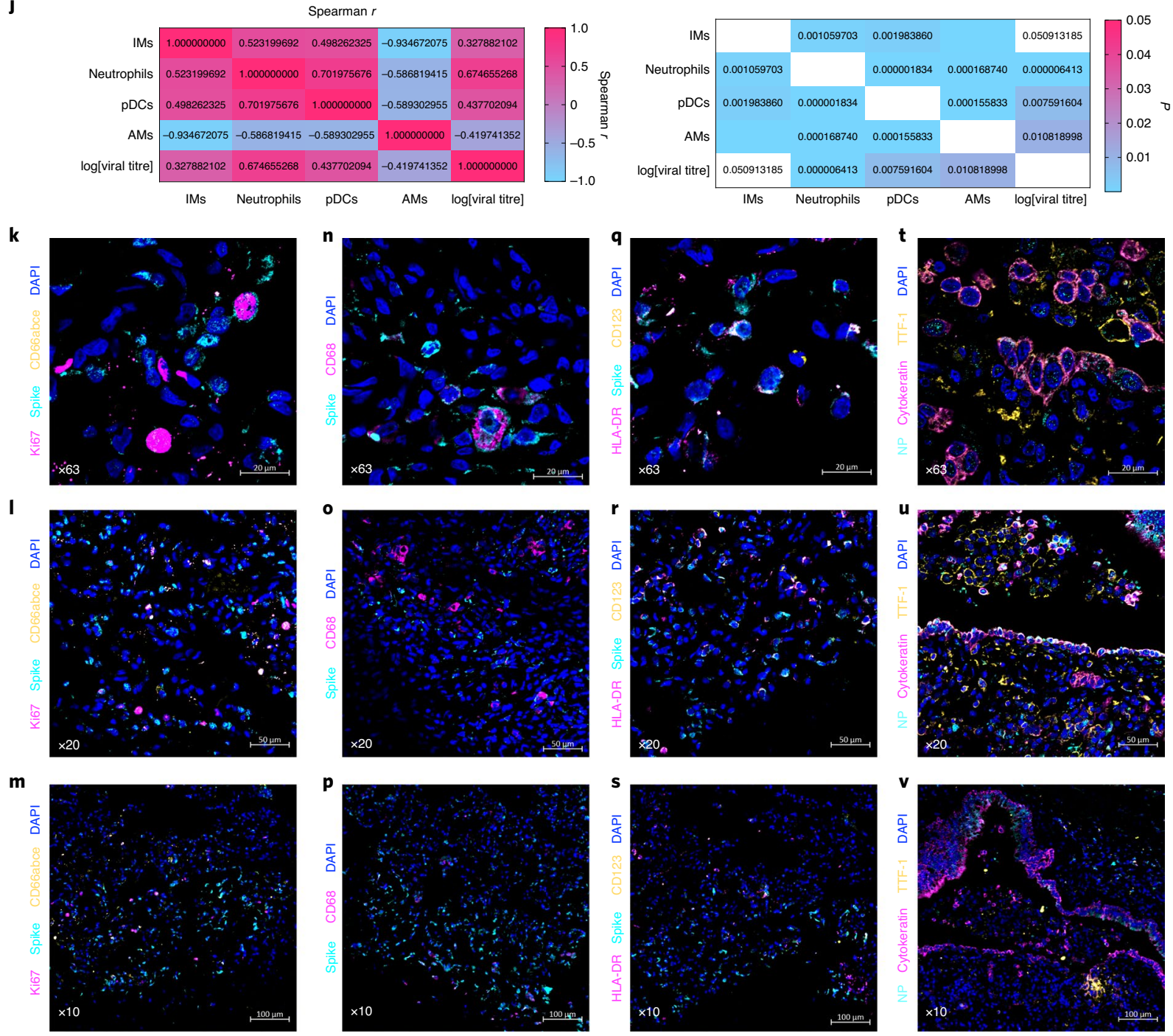

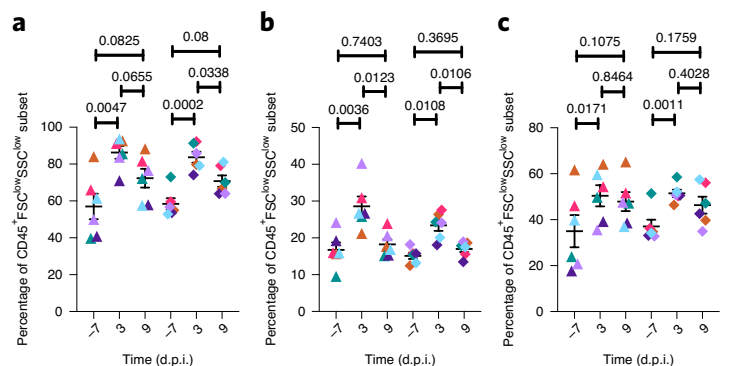

e

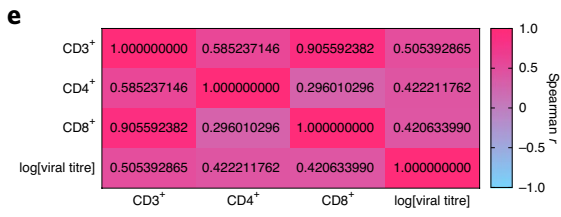

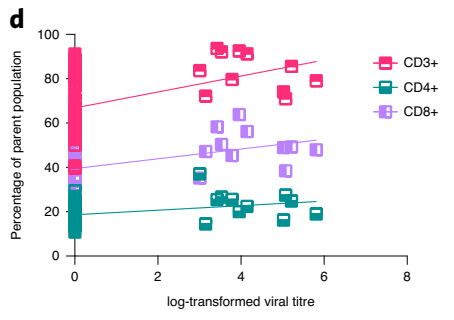

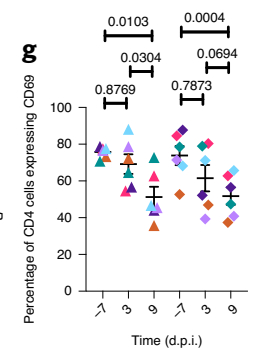

h

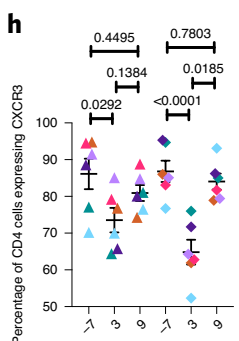

Time (d.p.i.)
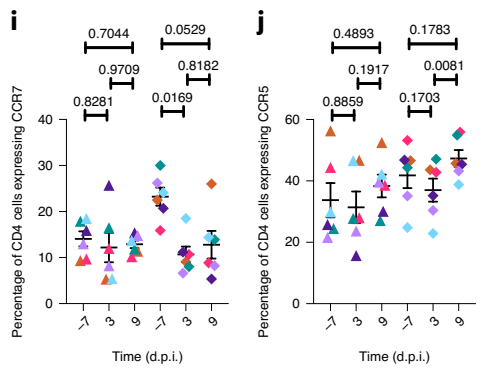

Time (d.p.i.)
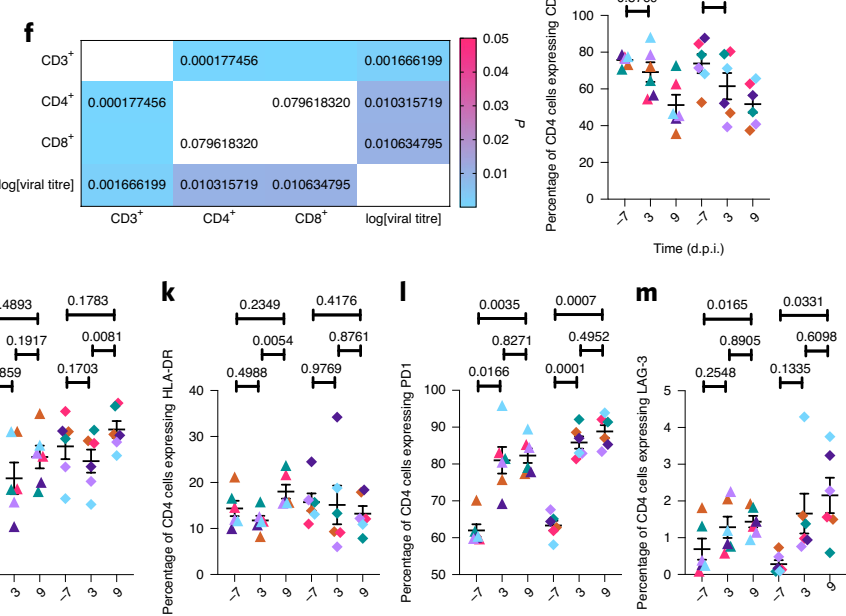

o

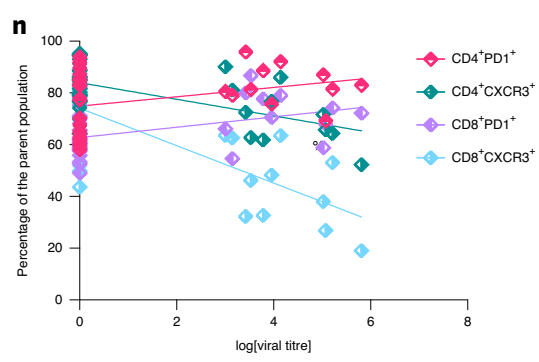

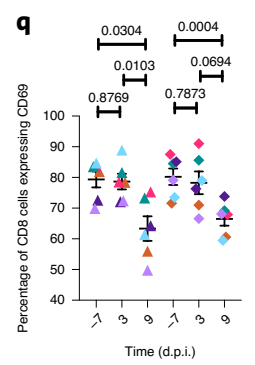

w

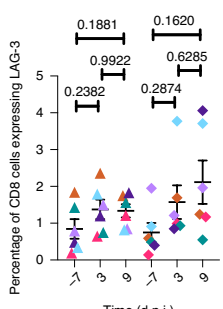

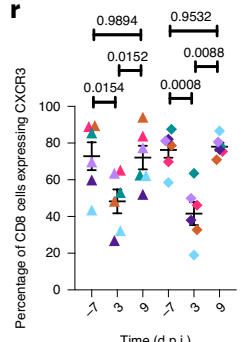

Time (d.p.i.)

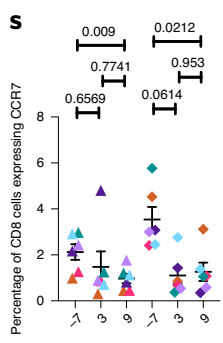

Time (d.p.i.)

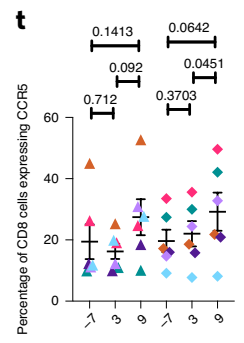

Time (d.p.i.)

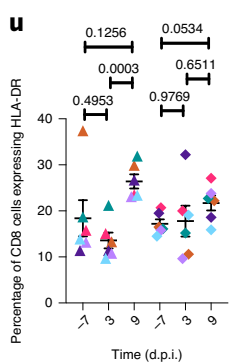

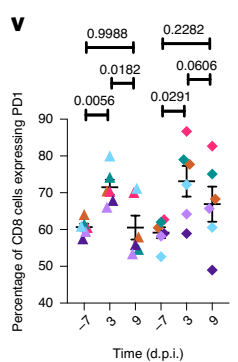

Fig. 5 | T cells in BAL of infected rhesus macaques. a-c, Frequencies of CD3+ $T$ cells (a), CD4+ $T$ cells $(\mathbf{b})$ and $C D 8^{+} T$ cells $(\mathbf{c})$ in BAL. d-f, Spearman's rank correlation of the cell types in a-c between the cellular fraction and the $\log _{10}$-transformed VRNA copy number in BAL was calculated (d) and the corresponding Spearman's rank correlation coefficient values (e) and $P$ values (f) are shown. $\mathbf{g}-\mathbf{m}, C D 4^{+}$- -cell subsets expressing the early activation marker CD69 (g), CXCR3 (h), the memory marker CCR7 (i), CCR5 (j), HLA-DR (k), PD-1 (I) and LAG-3 (m). n-p, Spearman's rank correlation between PD1 and CXCR3 expression on CD4 $4^{+}$and $C D 8^{+}$T cells and $\log _{10}$-transformed vRNA copy number in BAL was calculated (n) and the corresponding Spearman's rank correlation coefficient values (o) and $P$ values $(\mathbf{p})$ are shown. $\mathbf{q}-\mathbf{w}, C^{+} 8^{+}$T-cell subsets expressing the early activation marker $C D 69$ (q), CXCR3 (r), the memory marker CCR7 (s), CCR5 (t), HLA-DR (u), PD-1 (v) and LAG-3 (w). The shapes indicate old (triangles) and young (diamonds) animals. The different colours represent individual animals (Supplementary Table 1). $n=12$. For $\mathbf{a}-\mathbf{c}, \mathbf{g}-\mathbf{m}$ and $\mathbf{q}-\mathbf{w}$, data are mean \pm s.e.m. $n=12$. Statistical analysis was performed using two-way repeated-measures ANOVA with Geisser-Greenhouse correction for sphericity and Tukey post hoc correction for multiple testing (GraphPad Prism 8). For a-c, $\mathbf{g}-\mathbf{m}$ and $\mathbf{q}-\mathbf{w}, P$ values are indicated above the plots.

whereas CXCR3 expression was inversely correlated (Fig. 5n-p). There were no age-related differences in T-cell responses. Rapid influx of myeloid cells expressing type-I IFNs probably results in immune control of SARS-CoV-2 infection, but enables viral antigens to persist, which recruits $\mathrm{T}$ cells with a profile associated with immune activation in the effector phase. 

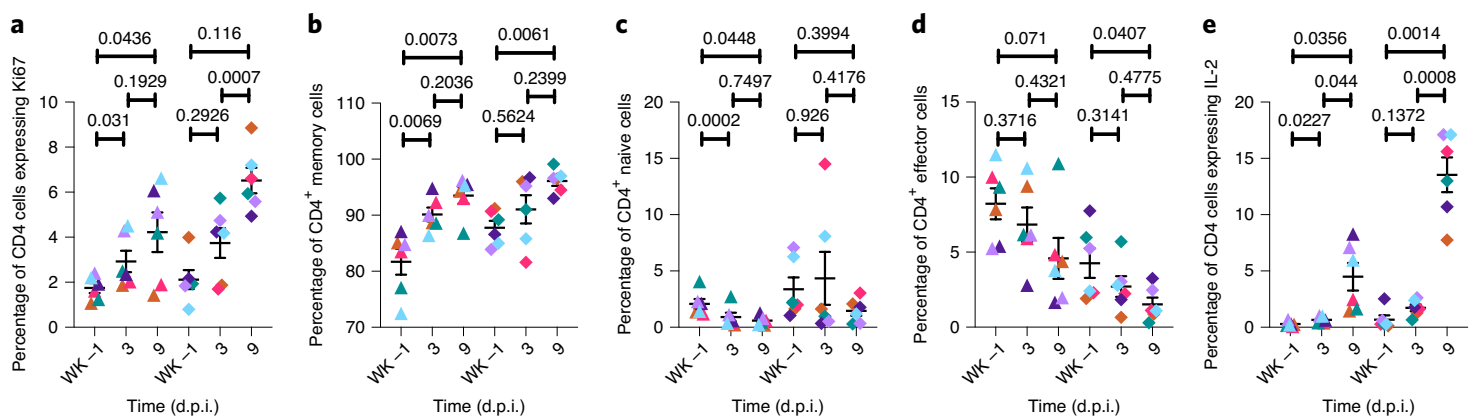

Time (d.p.i.)
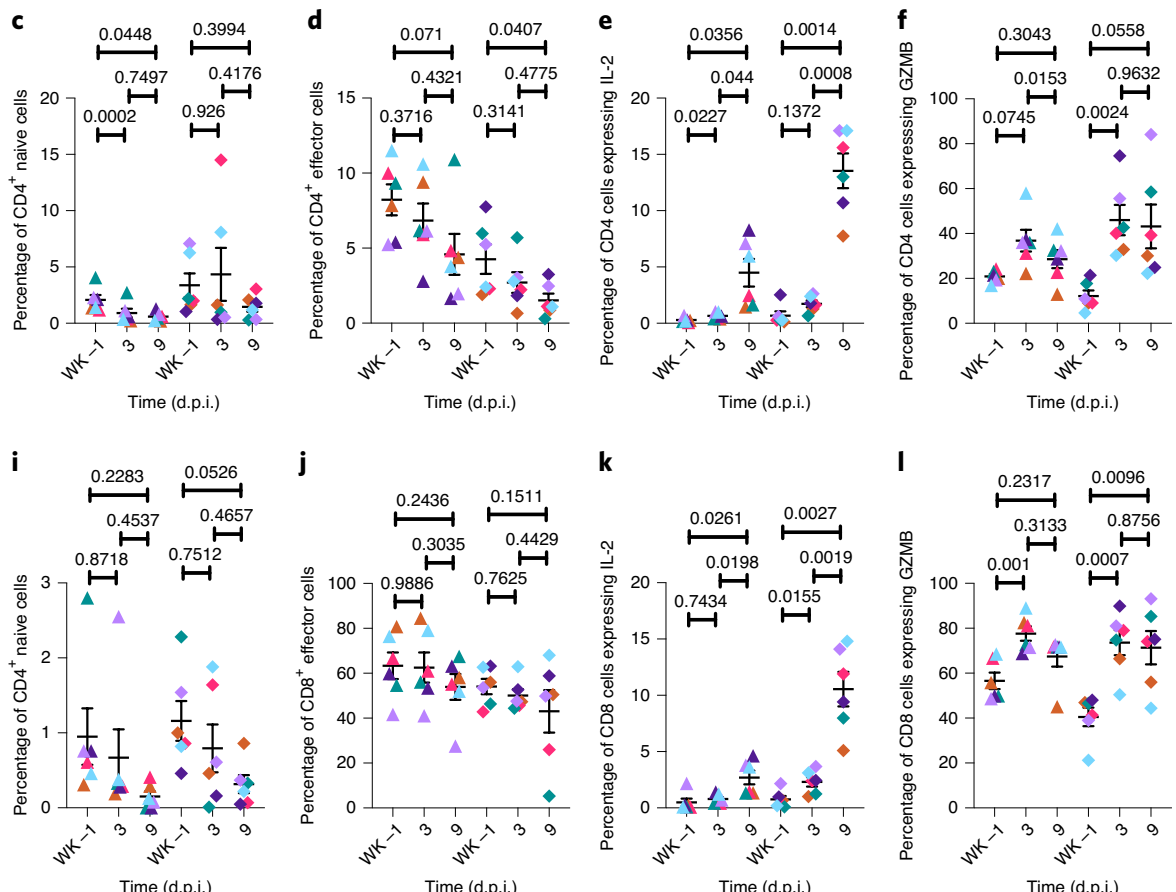

Time (d.p.i.)

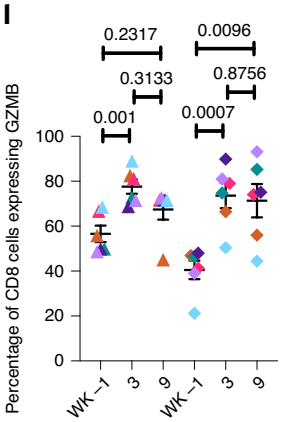

Time (d.p.i.)
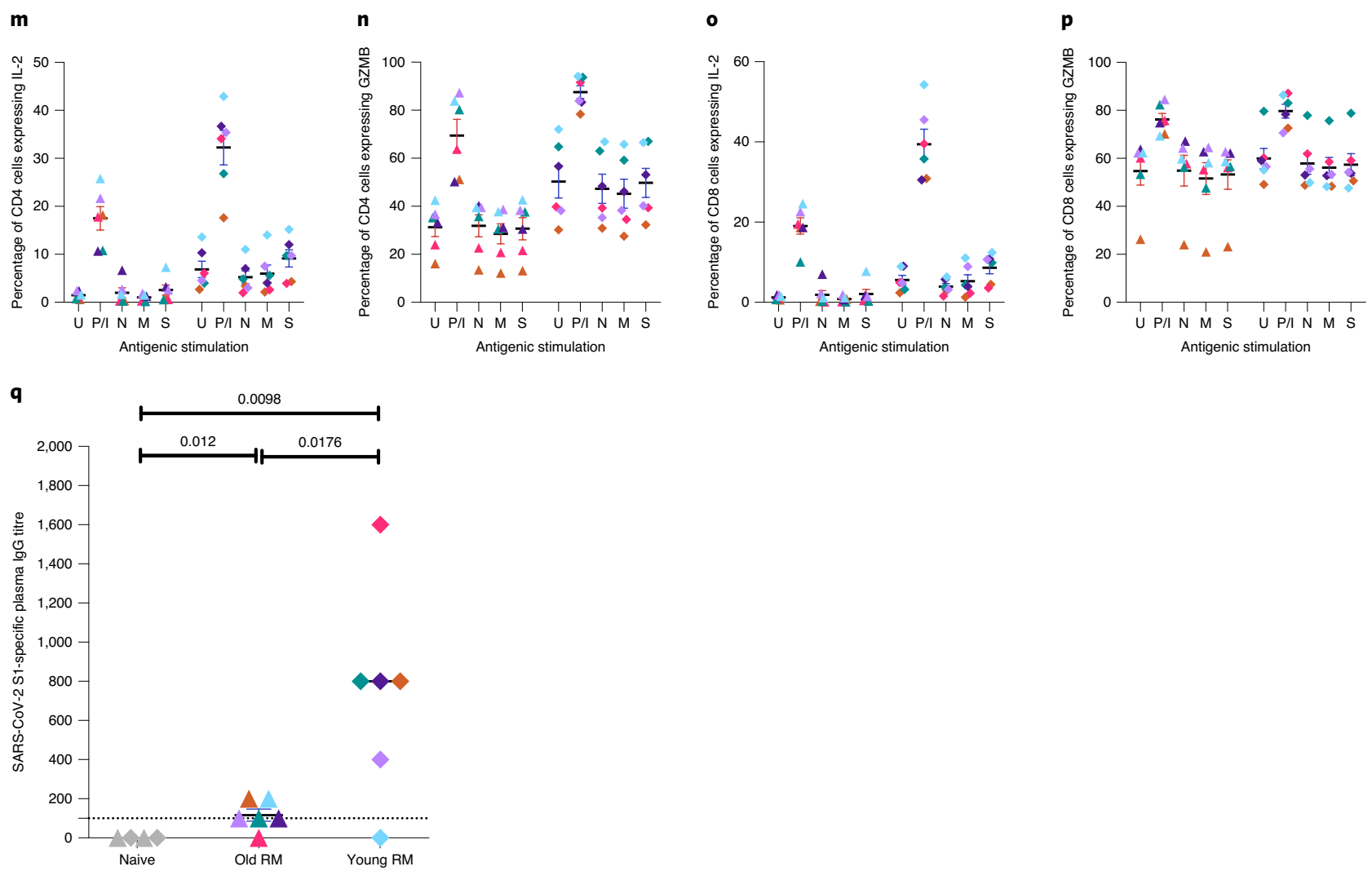

Fig. 6 | Memory T cells in BAL of infected rhesus macaques. a-f, Frequencies of the following CD4+ T-cell subsets: expressing Ki67 (a), memory (b), naive (c), effector (d), expressing IL-2 (e) and expressing GZMB (f) in BAL. g-I, Frequencies of the following CD8 ${ }^{+}$T-cell subsets: expressing Ki67 (g), memory (h), naive (i), effector (j), expressing IL-2 (k) and expressing GZMB (I). m-p, Cells from BAL were stimulated overnight (12-14h) with mock control (U), phorbol 12-myristate 13-acetate (PMA)-ionomycin (P/I) or SARS-CoV-2-specific peptide pools of the nucleocapsid (N), membrane (M) and spike (S) proteins. Antigen-specific cytokine secretion in T cells was estimated using flow cytometry. The fraction of CD4+ $4^{+}$cells secreting IL-2 (m) and GZMB (n); and CD8 ${ }^{+}$ T cells secreting IL-2 (o) and GZMB (p). The shapes indicate old (triangles) and young (diamonds) animals. The different colours represent individual animals (Supplementary Table 1). $n=12$. Statistical analysis was performed using two-way repeated-measures ANOVA with Geisser-Greenhouse correction for sphericity and Tukey post hoc correction for multiple testing (GraphPad Prism 8). q, The SARS-CoV-2 spike-protein-specific antibody titre in the plasma of rhesus macaques at the end point compared with the naive control. The shapes indicate old (triangles) and young (diamonds) animals. The different colours represent individual animals (Supplementary Table 1). $n=4$ (naive), $n=6$ (old) and $n=6$ (young). Statistical analysis was performed using ordinary one-way ANOVA with Dunnett's post hoc test (GraphPad Prism 8 ). For $\mathbf{a}-\mathbf{I}$ and $\mathbf{q}, P$ values are indicated above the plots. Data are mean \pm s.e.m. $(\mathbf{a}-\mathbf{q})$. 
To extrapolate from phenotype to function, we explored proliferation, immune mediator production and memory phenotypes. BAL $\mathrm{CD}^{+}$and $\mathrm{CD}^{+} \mathrm{T}$ cells exhibiting proliferative (Fig. 6a,g) and memory markers (Fig. 6b,h) were significantly increased after infection, whereas those expressing naive (Fig. $6 \mathrm{c}, \mathrm{i}$ ) and effector (Fig. $6 \mathrm{~d}, \mathrm{j}$ ) phenotypes were significantly reduced. The percentage of $\mathrm{CD}^{+}$(Fig. 6e) and $\mathrm{CD}^{+}$(Fig. 6k) T cells expressing IL-2 and granzyme B (GZMB; Fig. 6f,l) were significantly elevated. No significant effect of age was observed, although IL-2 expression on T cells was higher for young macaques compared with old macaques. These results suggest that the induction of robust $\mathrm{T}$-cell immune responses (both $\mathrm{CD}^{+}$and $\mathrm{CD}^{+} \mathrm{T}$ cells) is generated in the lungs (BAL) as early as day 3 and maintained at 9 d.p.i. in many cases. After ex vivo restimulation of $\mathrm{T}$ cells from BAL at 9 d.p.i. with $\mathrm{CoV}$-specific peptide pools, $\mathrm{CD}^{+}$and $\mathrm{CD}^{+} \mathrm{T}$ cells expressing IL-2, GZMB, IFN $\gamma$, IL-17 and TNF $\alpha$ were not significantly elevated beyond baseline values until day 12 (Fig. 6).

The immunophenotyping results were confirmed by studying cytokine production in BAL and plasma longitudinally ${ }^{19}$ (Extended Data Fig 10) and using spike-protein-specific antibodies at necropsy (Fig. 6q). IFN $\alpha$, IL-1Ra and IL-6 (Extended Data Fig. 10a-c) were elevated in BAL after infection, but levels rapidly normalized after the 3 d.p.i. peak. IFN $\alpha$ levels were also induced in plasma, but the levels of the other cytokines studied were not (Extended Data Fig. 8g). Overall, the longitudinal study immunological results were consistent with the acute infection study in the expression of type-I proinflammatory cytokines (IFN $\alpha$ ) and IL-6. The plasma of these macaques contained high levels of viral spike-protein-specific IgG at necropsy (Fig. 6q). The levels were significantly higher in young versus old animals. Altered antibody production, coupled with T-cell exhaustion, in the setting of chronic type-I IFN signalling has been reported earlier for other chronic viral infections ${ }^{20-22}$. In the face of chronic T-cell exhaustion, IL-10 responses may result in poor downstream antibody formation, as observed in our results.

As pulmonary pathology was significantly higher in baboons compared with in rhesus macaques (Fig. 1u), we studied the expression of various proinflammatory and protective cytokines and chemokines in BAL fluid derived from both young and old baboons. MIF, IL-6, CRP and IP-10 (Supplementary Fig. 4a-d) expression was significantly induced; the magnitude was greater in old animals (Supplementary Fig. 4a-d). BAL and serum levels of several other cytokines, chemokines and inflammatory markers were also increased (Supplementary Figs. 4e-m and 5), including IL1Ra, perforin and IL-8. Plasma IP-10, RANTES and IFN $\alpha$ levels were elevated (Supplementary Fig. 5a-d). The higher persistence of vRNA in some compartments (Fig. 1i-1) coupled with a greater severity of inflammation in the lungs (Fig. 1u) suggests that baboons, especially older ones, develop more-severe and longer-lasting disease than macaques, which is supported by the higher levels of proinflammatory cytokines in BAL of old baboons relative to young baboons (Supplementary Fig. 4a-d).

\section{Discussion}

Rhesus macaques, baboons and marmosets can all be infected with SARS-CoV-2 but show differential progression to COVID-19. Whereas older marmosets have a mild infection, macaques developed moderate progressive pneumonia that resolves, accompanied by a marked reduction in lung and nasal viral loads. Baboons have the most severe lung pathology, and the greatest viral load in RS. SARS-CoV-2 infection is associated with dynamic lung influxes of specific myeloid cell subsets, particularly IMs, neutrophils and pDCs, and viral proteins can be detected in these cells. This helps to explain the development of COVID-19 pneumonia and also the subsequent control through expression of a strong type-I IFN response. Control of infection is accompanied by resolution of viraemia and radiological lesions but viral antigens and remnant histopathological lesions persist over the course of two weeks. Integration of state-of-the-art CT scanning and innovative algorithms to assess the extent of lung involvement with viral loads in NS and BAL have enabled reproducible and quantifiable metrics of infection. Our experimental models have been useful for preclinical testing of candidate vaccines ${ }^{23}$ and therapies ${ }^{24}$ for COVID-19.

We were unable to detect replicative virus in the lungs using either plaque assays or subgenomic PCR after two weeks of infection, although live virus was readily recovered after 3 d.p.i. using both methods. However, various SARS-CoV-2 protein antigens were detected after two weeks in the tissues of NHPs, suggesting antigenic but not viral persistence. This is supported by the finding that the correlation between $\mathrm{PD}-1$ expression on $\mathrm{CD}^{+} \mathrm{T}$ cells with vRNA and a lack of induction of antigen-specific immune effector cytokine production by these cells might also be due to the comparatively shorter duration of this study, as longer studies have clearly established a protective T-cell immunity in SARS-CoV-2 infection ${ }^{25,26}$. Although there was viral clearance and improvement in radiological scores over the course of the study, the inflammation parameters studied and histology were more protracted resulting in a limited correlation between end-point histological and radiological scores. Furthermore, pathways related to angiogenesis and thrombosis are enriched in the lungs of infected macaques compared with healthy macaques ${ }^{9}$. Histopathology analysis was performed at 3 d.p.i. and 12/14 d.p.i.; we expect that radiologic lesions at 6 d.p.i. are a combination of the features described above.

As COVID-19 disproportionately affects older humans, we included age as an independent variable in our studies. Age-related effects were more pronounced in baboons than macaques. Baboons developed more-severe inflammatory lesions compared with macaques. Baboons are also a preferred model for cardiovascular and metabolic diseases including diabetes ${ }^{27-29}$; further development of the baboon model may therefore prove especially useful for the study of such comorbidities with COVID-19.

Age-related differences during infection in macaques was striking. Older animals generated substantially reduced amounts of SARS-CoV-2-specific antibodies compared with young macaques. Despite comparable viral replication and immune responses, the effect of age on neutralizing immunity may be a factor in the more pronounced COVID-19 disease in the elderly population. We propose that old macaques could be a useful model for testing therapies and vaccines for elderly humans.

\section{Methods}

Study approval. All of the infected animals were housed under Animal Biosafety Level 3 or 4 (ABSL3, ABSL4) facilities at the Southwest National Primate Research Center, where they were treated according to the standards recommended by AAALAC International and the NIH Guide for the Care and Use of Laboratory Animals. Sham controls were housed under ABSL2. The animal studies in each of the species were approved by the Animal Care and Use Committee of the Texas Biomedical Research Institute and as an omnibus Biosafety Committee protocol.

Animal studies and clinical evaluations. Sixteen (eight young and eight old; Supplementary Table 1) Indian-origin rhesus macaques (Macaca mulatta) and twelve (six young and six old) African-origin baboons (Papio hamadryas), all from the Southwest National Primate Research Center (SNPRC) breeding colonies, were exposed through multiple routes of inoculation (ocular, $100 \mu \mathrm{l}$; intranasal, $200 \mu$ l, using a MADgic Intranasal Mucosal Atomization Device (Teleflex); intratracheal, $200 \mu \mathrm{l}$, using a paediatric-size laryngo-tracheal Mucosal Atomization Device (Teleflex)) to $500 \mu \mathrm{l}$ of an undiluted stock of SARS-CoV-2, which had a titre of $2.1 \times 10^{6}$ p.f.u. per $\mathrm{ml}$, resulting in the administration of $1.05 \times 10^{6}$ p.f.u. SARS-CoV-2. SARS-CoV-2 generated from isolate USA-WA1/2020 was used for animal exposures. A fourth cell culture passage of SARS-CoV-2 was obtained from Biodefense and Emerging Infections Research Resources Repository (BEI Resources, NR-52281; GenBank: MN985325.1) and propagated at Texas Biomed. The stock virus was passaged for a fifth time in Vero E6 cells at a multiplicity of infection (m.o.i.) of approximately 0.001 . This master stock was used to generate a sixth cell culture passage exposure stock by infecting Vero E6 cells at a m.o.i. of 0.02 . The resulting stock had a titre of $2.10 \times 10^{6}$ p.f.u. per $\mathrm{ml}$ and was attributed the lot number 20200320 . The exposure stock was confirmed to be 
SARS-CoV-2 using deep sequencing and was identical to the published sequence (GenBank: MN985325) strain USA-WA1/2020 (BEI Resources, NR-52281). Six Brazilian-origin common marmosets (Callithrix jacchus) were also infected through the combined routes ( $80 \mu \mathrm{l}$ intranasal; $40 \mu \mathrm{l}$ ocular ( $20 \mu \mathrm{l}$ per eye); $40 \mu \mathrm{l}$ oral, performed twice for a total of $160 \mu \mathrm{l}$ intranasal, $80 \mu \mathrm{l}$ ocular, $80 \mu \mathrm{l}$ oral; and $100 \mu \mathrm{l}$ intratracheal once) of the same stock. The total target dose presented to marmosets was $8.82 \times 10^{5}$ p.f.u. per ml. We included sham-infected animals, which underwent all of the procedures (with the exception of necropsy) to control for the impact of multiple procedures over the course of this study (Supplementary Table 1). Four macaques, baboons and marmosets were each sham-infected with DMEM-10 medium (the storage vehicle of the virus) to be used as procedural controls. Infected animals were euthanized for tissue collection at necropsy, and control animals were returned to the colony. Macaques were enrolled from a specific-pathogen-free colony maintained at the SNPRC and were tested to be free from SPF-4 (simian retrovirus D, SIV, STLV-1 and herpes B virus). All of the animals, including the baboons and the marmosets, were also free of Mycobacterium tuberculosis. Animals were monitored regularly by a board-certified veterinary clinician for rectal body temperature, weight and physical examination. Collection of blood, BAL, nasal swab and urine, under tiletamine-zolazepam (Telazol) anaesthesia, was performed as described below, except that BAL was not performed in marmosets. Four macaques were sampled daily until euthanasia at 3 d.p.i. All of the other macaques and all of the baboons were sampled at $0,3,6,9$ and 12 d.p.i. and at euthanasia (BAL was performed weekly). Blood was collected for complete blood cell analysis and specialized serum chemistry analysis. Animals were observed daily to record alert clinical measurements. Nasal (longitudinal) or nasopharyngeal (acute) swabs and BALs were obtained to measure viral loads in a longitudinal manner, as described previously ${ }^{14}$. In brief, in a sitting position, the larynx was visualized and a sterile feeding tube was inserted into the trachea and advanced until it was met with resistance. Up to $80 \mathrm{ml}$ of warm sterile saline was instilled, divided into multiple aliquots. Fluid was aspirated and collected for analysis.

CXRs. Clinical radiographic evaluation was performed as follows: the lungs of all of the animals were imaged by conventional CXR as previously described ${ }^{30}$. Three-view thoracic radiographs (ventrodorsal, right and left lateral) were performed at all of the sampling time points. High-resolution computed tomography (CT) was performed daily over 3 d.p.i. in 4 infected macaques and on 6 and 12 d.p.i. in 3 young and 3 old macaques as described in the next section. Images were evaluated by a board-certified veterinary radiologist and scored as normal, mild moderate or severe disease. The changes were characterized as to location (lung lobe) and distribution (perivascular/peribronchial, hilar, peripheral, diffuse, multifocal/patchy).

CT imaging and quantitative analysis of lung pathology. The animals were anaesthetized using Telazol $\left(2-6 \mathrm{mg} \mathrm{kg}^{-1}\right)$ and maintained by inhaled isoflurane delivered through the Hallowell 2002 ventilator anaesthesia system (Hallowell). Animals were intubated to perform end-inspiratory breath-hold using a remote breath-hold switch. Lung field CT images were acquired using the Multiscan LFER150 PET/CT (MEDISO) scanner. Image analysis was performed using 3D ROI tools available in Vivoquant (Invicro). The percentage change in lung hyperdensity was calculated to quantify lung pathology ${ }^{1,2}$. The lung volume involved in pneumonia was quantified as follows. In brief, lung segmentation was performed using a connected thresholding feature to identify the lung ROI by classifying all of the input voxels of the scan in the range of $-850 \mathrm{HU}$ to $-500 \mathrm{HU}$. Smoothing filters were used to reassign every ROI voxel value to the mode of the surrounding region with defined voxel radius and iterations to reconstruct the lung ROI. Thereafter, global thresholding was applied to classify the voxels within the lung ROI in the range of $-490 \mathrm{HU}$ to $+500 \mathrm{HU}$ to obtain the lung hyperdensity ROI. The resultant ROIs were then rendered in the maximum intensity projection view using the VTK feature.

vRNA determination. vRNA from plasma/sera, BAL, urine, saliva, swabs (nasal/ nasopharyngeal, oropharyngeal, rectal) and lung homogenates was determined using quantitative PCR with reverse transcription (RT-qPCR) and vRNA isolation was performed as previously described for Middle East respiratory syndrome (MERS)-CoV and SARS-CoV ${ }^{12,27,28}$. RNA extraction from fluids was performed using the epMotion M5073c Liquid Handler (Eppendorf) and the NucleoMag Pathogen Kit (Macherey-Nagel). Test samples $(100 \mu \mathrm{l})$ were mixed with $150 \mu \mathrm{l}$ of $1 \times$ DPBS (Gibco) and $750 \mu$ TRIzol LS. Inactivation controls were prepared with each batch of samples to ensure that no cross contamination occurred during inactivation. Samples were thawed at room temperature and then-for serum, swabs and urine samples- $10 \mu$ g yeast tRNA was added, along with $1 \times 10^{3}$ p.f.u. of MS2 phage (Escherichia coli bacteriophage MS2, ATCC). DNA LoBind Tubes (Eppendorf) were prepared with $20 \mu \mathrm{l}$ of NucleoMag B-Beads (NucleoMag Pathogen Kit, Macherey-Nagel) and $975 \mu$ of buffer NPB2 (NucleoMag Pathogen Kit, Macherey-Nagel). After centrifugation, the upper aqueous phase of each sample was transferred to the corresponding new tube containing NucleoMag B-Beads and buffer NPB2. The samples were mixed using a HulaMixer (Thermo Fisher Scientific), rotating for $10 \mathrm{~min}$ at room temperature. The samples were then transferred to the sample rack on a epMotion M5073c Liquid Handler (Eppendorf) for further processing according to the NucleoMag Pathogen kit instructions. For vRNA determination from tissues, $100 \mathrm{mg}$ of tissue was homogenized in $1 \mathrm{ml}$ TRIzol Reagent (Invitrogen, Grand Island) with a Qiagen steel bead and Qiagen Stratagene TissueLyser. To detect infectious virus, in brief, tissues were homogenized $10 \%(\mathrm{w} / \mathrm{v})$ in viral transport medium using Polytron PT2100 tissue grinders (Kinematica). After low-speed centrifugation, the homogenates were frozen at $-70^{\circ} \mathrm{C}$ until they were inoculated on Vero E6 cell cultures in tenfold serial dilutions. The SARS-CoV-2 RT-qPCR was performed using a CDC-developed 2019-nCoV_N1 assay with the TaqPath 1-Step RT-qPCR Master Mix, CG (Thermo Fisher Scientific). The assays were performed on a QuantStudio 3 instrument (Applied Biosystems) with the following cycling parameters: hold stage, $2 \mathrm{~min}$ at $25^{\circ} \mathrm{C}, 15 \mathrm{~min}$ at $50^{\circ} \mathrm{C}, 2 \mathrm{~min}$ at $95^{\circ} \mathrm{C}$; PCR stage, $45 \mathrm{cycles}$ of $3 \mathrm{~s}$ at $95^{\circ} \mathrm{C}$ and $30 \mathrm{~s}$ at $60^{\circ} \mathrm{C}$. The primers and probe used were as follows: 2019-nCoV_N1-F: GACCCCAAAATCAGCGAAAT (500 nM); 2019-nCoV_N1-R: TCTGGTTACTGCCAGTTGAATCTG (500 nM); 2019-nCoV_N1-P FAM/MGB probe: ACCCCGCATTACGTTTGGTGGACC (125 nM).

RNA extraction for subgenomic vRNA determination using RT-qPCR. Samples were inactivated using TRIzol LS Isolation Reagent (Invitrogen) as follows: $250 \mu \mathrm{l}$ of test sample was mixed with $750 \mu \mathrm{l}$ TRIzol LS. Inactivation controls were prepared with each batch of samples. Before extraction, $1 \times 10^{3}$ p.f.u. of MS2 phage (E. coli bacteriophage MS2, ATCC) was added to each sample to assess extraction efficiency. RNA extraction was performed using the epMotion M5073c Liquid Handler (Eppendorf) and the NucleoMag Pathogen kit (Macherey-Nagel). Extraction controls were prepared with each batch of samples. After processing, the presence of the eluate was confirmed and the extracted RNA was stored at $-80 \pm 10^{\circ} \mathrm{C}$.

\section{Determination of viral load using RT-qPCR. RNA samples $(5 \mu \mathrm{l})$ were} reacted using duplex RT-qPCR to detect both SARS-CoV-2 and MS2 phage. Two assays were used to assess the presence of SARS-CoV-2 in the samples. The CDC-developed 2019-nCoV_N1 assay was used to target a region of the $N$ gene. SARS-CoV-2_N1 probe (ACCCCGCATTACGTTTGGTGGACC) was labelled with 6-FAM fluorescent dye. The forward primer sequence was GACCCCAAAATCAGCGAAAT and the reverse primer sequence was TCTGGTTACTGCCAGTTGAATCTG. A secondary qPCR assay to measure subgenomic RNA was also performed to target a region of $E$ (envelope $)^{31,32}$. The probe was also labelled with 6-FAM fluorescent dye (ACACTAGCCATCCTTACTGCGCTTCG). The forward primer sequence was CGATCTCTTGTAGATCTGTTCTC and the reverse primer sequence was ATATTGCAGCAGTACGCACACA. The MS2 probe was labelled with VIC fluorescent dye. Both assays used the TaqPath 1-Step RT-qPCR Master Mix, CG (Thermo Fisher Scientific) and were performed using a QuantStudio 3 instrument (Applied Biosystems). The QuantStudio design and analysis software (Applied Biosystems) was used to run and analyse the results. Cycling parameters were set as follows: hold stage, $2 \mathrm{~min}$ at $25^{\circ} \mathrm{C}, 15 \mathrm{~min}$ at $50^{\circ} \mathrm{C}, 2 \mathrm{~min}$ at $95^{\circ} \mathrm{C}$; PCR stage: 45 cycles (N1 assay) or 40 cycles (E assay) of $3 \mathrm{~s}$ at $95^{\circ} \mathrm{C}$ and $30 \mathrm{~s}$ at $60^{\circ} \mathrm{C}$. The average $C_{\mathrm{t}}$ value for MS2 phage was calculated for all of the processed samples and SARS-CoV-2 quantification was performed only in samples in which the MS2 $C_{\mathrm{t}}$ value was lower than average MS2 $+5 \%$.

Pathology. Animals were euthanized and complete necropsy was performed. Gross images (lungs, spleen, liver) and organ weights (lymph nodes, tonsils, spleen, lungs, liver, adrenal glands) were obtained at necropsy. Representative samples of lung lymph nodes (inguinal, axillary, mandibular and mediastinal), tonsils, thyroid gland, trachea, heart, spleen, liver, kidneys, adrenal glands, digestive system (stomach, duodenum, jejunum, ileum, colon and rectum), testes or ovary, brain, eyes, nasal tissue and skin were collected for all animals. Tissues were fixed in $10 \%$ neutral-buffered formalin, processed to paraffin, sectioned at a thickness of $5 \mu \mathrm{m}$, stained with haematoxylin and eosin using standard methods, and evaluated by a board-certified veterinary pathologist.

\section{Tissue processing, flow cytometry, multiplex cytokine analyses,} immunohistochemistry, multicolour confocal microscopy and SARS-CoV-2 specific-antibody response detection for immune evaluations. Flow cytometry was performed as previously described ${ }^{14,33}$ on blood and BAL samples collected on days $3,6,9$ and 12, and at the end point, which occurred at 14-17 d.p.i. for various animals. A comprehensive list of antibodies used in these experiments is provided in Supplementary Table 8. For evaluations of peripheral blood, peripheral blood mononuclear cells were prepared as previously described. In brief, Cellular phenotypes were studied using the following antibodies: anti-CD3 (SP34-2), anti-CD4 (L200), anti-CD69 (FN50), anti-CD20 (2H7), anti-CD95 (DX2), anti-Ki67 (B56), anti-CCR5 (3A9), anti-CCR7 (3D12), anti-CD28 (CD28.2), anti-CD45 (D058-1283), anti-CXCR3 (1C6/CXCR3), anti-HLA-DR (L243), anti-CCR6 (11A9), anti-LAG-3 (polyclonal, R\&D Systems), anti-CD123 (7G3), anti-CD14 (M5E2), anti-CD206 (206), anti-CD16 (3G8), anti-CD163 (GHI/61), anti-CD66abce (TET2, Miltenyi Biotech), anti-CD40 (5C3), anti-IL-2 (MQ1$17 \mathrm{H} 12)$, anti-GZMB (GB11), which were all purchased from BD Biosciences unless 
specified. Anti-CD8 (RPA-T8), anti-CD11c (3.9), anti-TNF $\alpha$ (MAb11), anti-IFN $\gamma$ (B27), anti-IL-17 (BL168) and anti-PD-1 (EH12.2H7) antibodies were purchased from BioLegend. For antigenic stimulation, cells were cultured overnight with SARS-CoV-2-specific peptide pools of the nucleocapsid, membrane and spike proteins (PepTivator SARS-CoV-2 peptide pool, Miltenyi Biotech). A description of the detailed gating strategy for the detection and enumeration of various cellular phenotypes is provided in Supplementary Fig. 6.

Immunohistochemistry was performed on sections (thickness, $4 \mu \mathrm{m}$ ) of lung, nasal cavity and tonsils. The sections were baked at $65^{\circ} \mathrm{C}$ for $30 \mathrm{~min}$ followed by deparaffinization using xylene and subsequent hydration with decreasing gradations of ethanol as described previously ${ }^{14,34}$. Heat-induced antigen retrieval was performed using sodium citrate buffer ( $10 \mathrm{mM}, \mathrm{pH} 6.0)$ followed by blocking ( $3 \% \mathrm{BSA}$ in TBST for $1 \mathrm{~h}$ at $37^{\circ} \mathrm{C}$ ). For SARS-CoV-2 detection, specimens were incubated with rabbit anti-SARS-CoV-2 spike antibodies (ProSci, $1: 200,37^{\circ} \mathrm{C}$ for $2 \mathrm{~h}$ ) or anti-SARS-CoV-2 nucleocapsid antibodies (Sino Biologicals, 1:100, $2 \mathrm{~h}$ at $37^{\circ} \mathrm{C}$ ). Anti-human-ACE2 antibodies (R\&D Systems, 1:50, $2 \mathrm{~h}$ at $37^{\circ} \mathrm{C}$ ) were used to identify ACE2. Mouse anti-human-CD66abce PE-conjugated antibodies (Miltenyi Biotech, $1: 20,2 \mathrm{~h}$ at $37^{\circ} \mathrm{C}$ ) were used to identify neutrophils; mouse anti-CD68 antibodies (Thermo Fisher Scientific, $1: 100,2 \mathrm{~h}$ at $37^{\circ} \mathrm{C}$ ) for macrophages; and pDCs were identified by costaining with PE-conjugated mouse anti-human-CD123 (BD Biosciences, $1: 20,37^{\circ} \mathrm{C}$ for $2 \mathrm{~h}$ ) and mouse anti-human-HLA-DR (Thermo Fisher Scientific, 1:100, $2 \mathrm{~h}$ at $37^{\circ} \mathrm{C}$ ) antibodies. Furthermore, mouse anti-Ki67 antibodies (BD Biosciences, $1: 50,2 \mathrm{~h}$ at $37^{\circ} \mathrm{C}$ ) were used to detect actively proliferating cells. Pan-cytokeratin mouse monoclonal antibodies (AE1/AE3) Alexa Fluor 488 (Thermo Fisher Scientific, $1: 50,2 \mathrm{~h}$ at $37^{\circ} \mathrm{C}$ ) were used to detect type-1 pneumocytes and epithelial cells, and anti-TTF-1 mouse monoclonal antibodies (Thermo Fisher Scientific, 1:200, $2 \mathrm{~h}$ at $37^{\circ} \mathrm{C}$ ) were used to detect type-2 pneumocytes. Chicken anti-rabbit IgG $(\mathrm{H}+\mathrm{L})$ Alexa Fluor 488 conjugate; goat anti-mouse IgG $(\mathrm{H}+\mathrm{L})$ Alexa Fluor 647 conjugate; donkey anti-mouse IgG $(\mathrm{H}+\mathrm{L})$ Alexa-Fluor 555 conjugate; donkey anti-goat IgG $(\mathrm{H}+\mathrm{L})$ Alexa Fluor 555; goat anti-mouse IgG1 secondary antibody Alexa Fluor 555 (Thermo Fisher Scientific, $1: 400,1 \mathrm{~h}$ at $37^{\circ} \mathrm{C}$ ) were used to label spike and nucleocapsid, Ki67 and HLA-DR, CD68, ACE2 and TTF-1 primary antibodies, respectively. Tissue sections were then stained with DAPI (Thermo Fisher Scientific, $1: 5,000,5 \mathrm{~min}$ at $37^{\circ} \mathrm{C}$ ) with subsequent mounting using Prolong Diamond Antifade mountant (Thermo Fisher Scientific). A Ziess LSM 800 confocal microscope was used to visualize the stained sections ( $\times 10, \times 20$ and $\times 63$ magnification). RNA was isolated for $\mathrm{qPCR}$ and data were analysed as described previously $y^{19}$

The antibody response against SARS-CoV-2 was measured using an enzyme-linked immunosorbent assay (ELISA) using the SARS-CoV-2 spike S1 subunit protein (Sino Biological) as the capture antigen. Each well of a 96-well microtitre plate (Corning, 2592) was coated with $100 \mathrm{ng}$ of protein in $100 \mu \mathrm{l}$ carbonate-bicarbonate buffer $(\mathrm{pH} 9.6)$ and incubated overnight at $4{ }^{\circ} \mathrm{C}$. The next day, the plate was washed twice with $1 \times$ PBS containing $0.05 \%$ Tween-20 (PBST) followed by blocking with $5 \%$ non-fat dry milk in PBST at room temperature for $2 \mathrm{~h}$. Twofold serially diluted heat-inactivated plasma from infected and control macaques was added to duplicate wells and incubated at $37^{\circ} \mathrm{C}$ for $1 \mathrm{~h}$. After five washes with $1 \times$ PBST, $100 \mu$ of rabbit anti-monkey IgG peroxidase-conjugated antibodies (1:5,000 dilution in blocking buffer) was added to each well and incubated at $37^{\circ} \mathrm{C}$ for $1 \mathrm{~h}$. The plate was washed five times with $1 \times$ PBST. SureBlue TMB peroxidase substrate $(100 \mu \mathrm{l})$ was then added to each well and incubated at room temperature for $10 \mathrm{~min}$. The reaction was stopped by adding $100 \mu \mathrm{lTMB}$ stop solution and the plate was immediately read at $450 \mathrm{~nm}$. The end-point ELISA titre of binding antibodies was defined as the reciprocal of the serum dilution that resulted in a positive optical density reading, which is at least two times the mean optical density reading with no plasma control wells. The detection limit of the ELISA was considered to be the starting dilution (1:100) of the test sera.

Statistical analyses. Graphs were prepared and statistical comparisons were applied using GraphPad Prism v.8. Various statistical comparisons were performed as follows. Two-tailed Student's $t$-tests, ordinary ANOVA or one-way or two-way repeated measure ANOVA with Geisser-Greenhouse correction for sphericity and Tukey post hoc correction for multiple testing (GraphPad Prism 8) was applied where applicable and as described in the figure legends. For correlation analysis, Spearman's rank tests were applied. Statistical differences between groups were reported to be significant when the $P$ value was less than or equal to 0.05 . Data are presented as mean \pm s.e.m.

Reporting Summary. Further information on research design is available in the Nature Research Reporting Summary linked to this article.

\section{Data availability}

All data supporting the findings of this study are available within this manuscript and its Supplementary Information. Any additional data can be requested from the corresponding authors upon reasonable request.

Received: 14 July 2020; Accepted: 23 November 2020; Published online: 18 December 2020

\section{References}

1. Rockx, B. et al. Comparative pathogenesis of COVID-19, MERS, and SARS in a nonhuman primate model. Science https://doi.org/10.1126/science. abb7314 (2020).

2. Munster, V. J. et al. Respiratory disease in rhesus macaques inoculated with SARS-CoV-2. Nature https://doi.org/10.1038/s41586-020-2324-7 (2020)

3. $\mathrm{Yu}$, J. et al. DNA vaccine protection against SARS-CoV-2 in rhesus macaques. Science https://doi.org/10.1126/science.abc6284 (2020).

4. Chandrashekar, A. et al. SARS-CoV-2 infection protects against rechallenge in rhesus macaques. Science https://doi.org/10.1126/science.abc4776 (2020).

5. Lu, S. et al. Comparison of nonhuman primates identified the suitable model for COVID-19. Signal Transduct. Target Ther. 5, 157 (2020).

6. Blair, R. V. et al. Acute respiratory distress in aged, SARS-CoV-2 infected African green monkeys but not rhesus macaques. Am. J. Pathol. https://doi. org/10.1016/j.ajpath.2020.10.016 (2020).

7. Hoffmann, M. et al. SARS-CoV-2 cell entry depends on ACE2 and TMPRSS2 and is blocked by a clinically proven protease inhibitor. Cell 181, 271-280 (2020).

8. Matsuyama, S. et al. Enhanced isolation of SARS-CoV-2 by TMPRSS2-expressing cells. Proc. Natl Acad. Sci. USA 117, 7001-7003 (2020).

9. Rosa, B. A. et al. IFN signaling and neutrophil degranulation transcriptional signatures are induced during SARS-CoV-2 infection. Preprint at bioRxiv https://doi.org/10.1101/2020.08.06.239798 (2020).

10. Cockrell, A. S. et al. A spike-modified Middle East respiratory syndrome coronavirus (MERS-CoV) infectious clone elicits mild respiratory disease in infected rhesus macaques. Sci. Rep. 8, 10727 (2018).

11. Mantlo, E., Bukreyeva, N., Maruyama, J., Paessler, S. \& Huang, C. Antiviral activities of type I interferons to SARS-CoV-2 infection. Antivir. Res. 179, 104811 (2020).

12. Nile, S. H. et al. COVID-19: pathogenesis, cytokine storm and therapeutic potential of interferons. Cytokine Growth Factor Rev. https://doi.org/10.1016/j. cytogfr.2020.05.002 (2020).

13. Cai, Y. et al. In vivo characterization of alveolar and interstitial lung macrophages in rhesus macaques: implications for understanding lung disease in humans. J. Immunol. 192, 2821-2829 (2014).

14. Bucsan, A. N. et al. Mechanisms of reactivation of latent tuberculosis infection due to SIV co-infection. J. Clin. Invest. https://doi.org/10.1172/ JCI125810 (2019)

15. Gautam, U. S. et al. In vivo inhibition of tryptophan catabolism reorganizes the tuberculoma and augments immune-mediated control of Mycobacterium tuberculosis. Proc. Natl Acad. Sci. USA 115, E62-E71 (2018).

16. Munster, V. J. et al. Respiratory disease in rhesus macaques inoculated with SARS-CoV-2. Nature 585, 268-272 (2020).

17. Schaefer, I. M. et al. In situ detection of SARS-CoV-2 in lungs and airways of patients with COVID-19. Mod. Pathol. 33, 2104-2114 (2020).

18. Kuroda, M. J. et al. High turnover of tissue macrophages contributes to tuberculosis reactivation in simian immunodeficiency virus-infected rhesus macaques. J. Infect. Dis. https://doi.org/10.1093/infdis/jix625 (2018).

19. Ahmed, M. et al. Immune correlates of tuberculosis disease and risk translate across species. Sci. Transl. Med. 12, https://doi.org/10.1126/scitranslmed. aav0233 (2020).

20. Charles, E. D. \& Dustin, L. B. Hepatitis C virus-induced cryoglobulinemia. Kidney Int. 76, 818-824 (2009).

21. Haas, A., Zimmermann, K. \& Oxenius, A. Antigen-dependent and -independent mechanisms of $\mathrm{T}$ and $\mathrm{B}$ cell hyperactivation during chronic HIV-1 infection. J. Virol. 85, 12102-12113 (2011).

22. Hunziker, L. et al. Hypergammaglobulinemia and autoantibody induction mechanisms in viral infections. Nat. Immunol. 4, 343-349 (2003).

23. Vogel, A. B. et al. A prefusion SARS-CoV-2 spike RNA vaccine is highly immunogenic and prevents lung infection in non-human primates. Preprint at bioRxiv https://doi.org/10.1101/2020.09.08.280818 (2020).

24. Baum, A. et al. REGN-COV2 antibodies prevent and treat SARS-CoV-2 infection in rhesus macaques and hamsters. Science 370, 1110-1115 (2020).

25 . Deng, W. et al. Primary exposure to SARS-CoV-2 protects against reinfection in rhesus macaques. Science 369, 818-823 (2020).

26. Chandrashekar, A. et al. SARS-CoV-2 infection protects against rechallenge in rhesus macaques. Science 369, 812-817 (2020).

27. Cox, L. A. et al. Nonhuman primates and translational researchcardiovascular disease. ILAR J. 58, 235-250 (2017).

28. Rincon-Choles, H. et al. Renal histopathology of a baboon model with type 2 diabetes. Toxicol. Pathol. 40, 1020-1030 (2012).

29. Cole, S. A., Laviada-Molina, H. A., Serres-Perales, J. M., Rodriguez-Ayala, E. \& Bastarrachea, R. A. The COVID-19 pandemic during the time of the diabetes pandemic: likely fraternal twins? Pathogens https://doi.org/10.3390/ pathogens 9050389 (2020).

30. Kaushal, D. et al. Mucosal vaccination with attenuated Mycobacterium tuberculosis induces strong central memory responses and protects against tuberculosis. Nat. Commun. 6, 8533 (2015). 
31. Wolfel, R. et al. Virological assessment of hospitalized patients with COVID-2019. Nature 581, 465-469 (2020).

32. Corman, V. M. et al. Detection of 2019 novel coronavirus (2019-nCoV) by real-time RT-PCR. Euro Surveill. https://doi.org/10.2807/1560-7917. ES.2020.25.3.2000045 (2020).

33. Ganatra, S. R. et al. Anti-retroviral therapy does not reduce tuberculosis reactivation in a tuberculosis-HIV co-infection model. J. Clin. Invest. https:// doi.org/10.1172/JCI136502 (2020).

34. Mehra, S. et al. Granuloma correlates of protection against tuberculosis and mechanisms of immune modulation by Mycobacterium tuberculosis. J. Infect. Dis. 207, 1115-1127 (2013).

\section{Acknowledgements}

We acknowledge the work by our SNPRC veterinary technical and care staff (especially the veterinary technical/animal care groups headed by T. Camp, W. Hodgkins, M. Aguilar, D. Vandenberg and L. Rumpf) as well as the entire SNPRC and Texas Biomedical Research Institute administrative staff, especially $\mathrm{H}$. Hawn, for assistance with this study. This work was primarily supported by a philanthropic award to Texas Biomed Coronavirus Working Group; a SNPRC Pilot study award to L.D.G., R.C. Jr,

J.L.P., L.M.-S. and J.B.T.; and an award to R.C. Jr from Regeneron Pharmaceuticals (no. 2020_004110). This work was supported in part by federal funds from the Department of Health and Human Services; Office of the Assistant Secretary for Preparedness and Response; Biomedical Advanced Research and Development Authority (no. HHSO100201700020C); institutional NIH awards (P51OD111033 and U42OD010442); and a supplemental award (R01AI134236-04; principal investigator, S.A.K.)/COVID grant (no. 3R01AI134236-04W). The views expressed here are those of the authors and do not necessarily represent the views or official position of the funding agencies.

\section{Author contributions}

D.K.S. was responsible for conducting experiments, acquisition of data, analysis, putting the entire analysis together and helped to write the paper. S.R.G., B.S., M. Gazi., J. Cole., R.T., K.J.A., E.C., O.G., R.N.P., M.A., B.R., S.A.K. and M.M. conducted experiments, acquired and analysed some parts of data, and helped to write specific parts of the paper. R.E., T.-H.L., A.C., Y.G.-G., R.S., M. Gough., C.A., A. Blakley., J.F., C.B., H.S.,
L.P., J. Callery., A.M., B.K., P.E., V.H., J.S., S.G., A.G.V., A.O.-F., A.S., A.O., D.K.A., R.C., A. Baum., C.K., X.A. and A.K.V. conducted experiments. A.G., J.D., S.H.-U., P.A.F. and K.B. provided veterinary medicine expertise. C.N.R., K.S. and C.C. provided colony management expertise. T.J.C.A. provided data analysis expertise. L.M.-S. and J.L.P. provided virology expertise. J.T. and J.B.T. provided immunology expertise and helped to write the paper. E.J.D. Jr provided veterinary pathology expertise. C.H. and L.S.S. were responsible for funding, and L.S.S. provided immunology expertise and helped to write the paper. L.D.G. was responsible for funding, provided immunology expertise, oversaw baboon experiments and helped to write the manuscript. R.C. Jr was responsible for funding, provided molecular expertise, oversaw marmoset experiments and helped to write the manuscript. D.K. was responsible for the overall conduct of the experiment, including the rhesus model, funding, provided imaging and immunology expertise, and wrote the manuscript.

\section{Competing interests}

R.C. Jr is funded by Regeneron Inc., and A.O., D.K.A., R.C., A. Baum and C.K. are employed by Regeneron. However, this funder had no role in the design and execution of the experiments and the interpretation of data. The other authors declare no other competing interests.

\section{Additional information}

Extended data is available for this paper at https://doi.org/10.1038/s41564-020-00841-4. Supplementary information is available for this paper at https://doi.org/10.1038/ s41564-020-00841-4.

Correspondence and requests for materials should be addressed to L.D.G., R.C. or D.K.

Peer review information: Nature Microbiology thanks Robert Garry and the other, anonymous, reviewer(s) for their contribution to the peer review of this work.

Reprints and permissions information is available at www.nature.com/reprints. Publisher's note Springer Nature remains neutral with regard to jurisdictional claims in published maps and institutional affiliations.

(c) The Author(s), under exclusive licence to Springer Nature Limited 2020, corrected publication 2021 

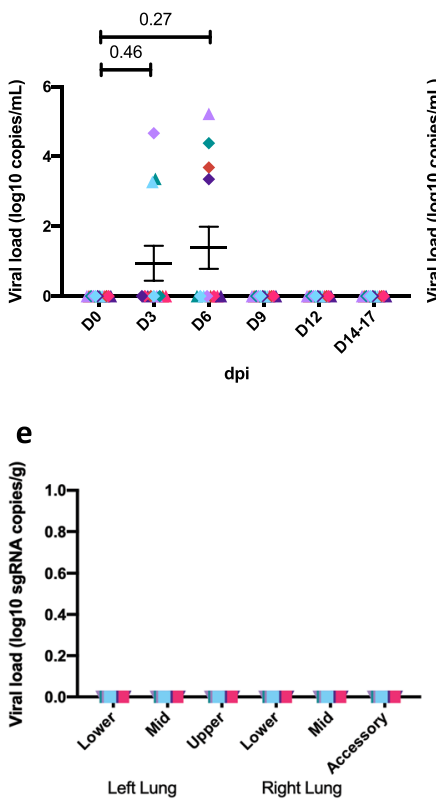

b

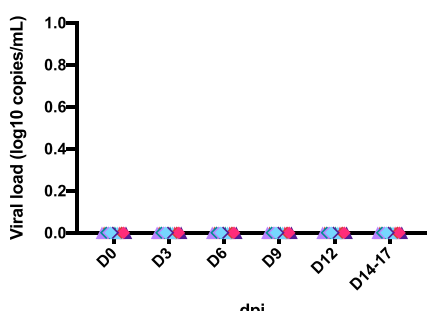

dpi

f

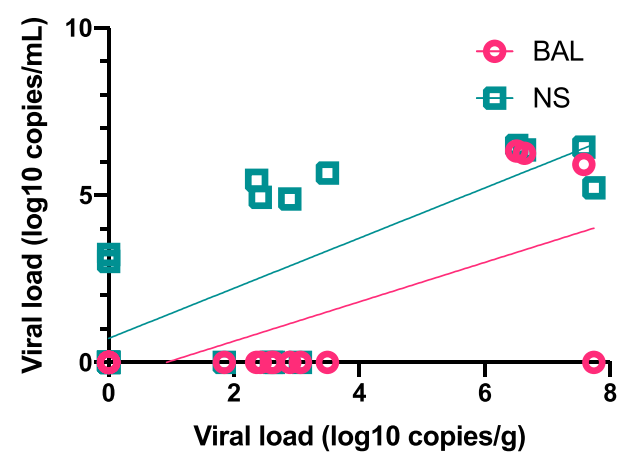

c

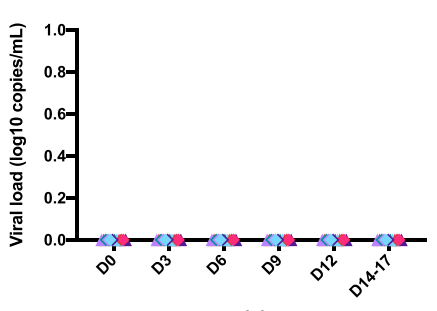

dpi

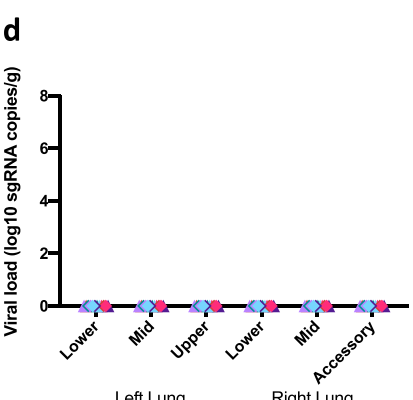

Left Lung

Right Lung

$\mathbf{g}$

Spearman $r$

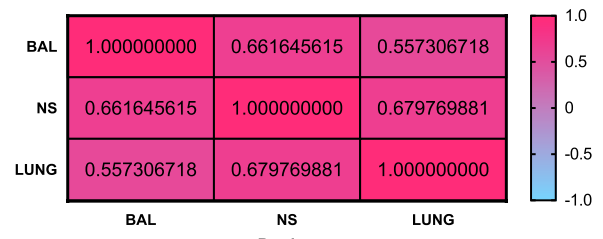

h

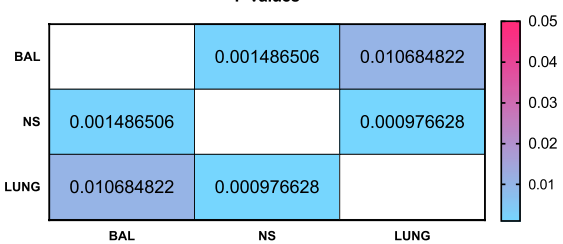

Extended Data Fig. 1 | Longitudinal viral RNA determination following SARS-CoV-2 infection in rhesus macaques. Viral RNA (log 10 copies/mL measured by RT-PCR in buccopharyngeal swab (a), plasma (b) and urine (c) of rhesus macaques longitudinally. Subgenomic viral RNA (log ${ }_{10}$ copies/gram of lung tissue was measured at endpoint in rhesus macaques and (d) and Baboons (e). (Rhesus: Old-Triangle, Young-Diamonds; Baboon: Old-Inverted triangle, Young-Square, Colors represent individual animals, Supplementary Table 1). $(n=12)$. Data are represented as mean \pm SEM. Two way Repeated-measures ANOVA with Geisser-Greenhouse correction for sphericity and Tukey's post hoc correction for multiple-testing (GraphPad Prism 8) was applied.

Correlations with Spearman's rank test between Log10 viral RNA copy number in Lung with BAL and NS (f) and corresponding values for Spearman's rank correlation coefficient (g) and P values (h). Coloring scheme for $\mathbf{f}-$ BAL (magenta circle), NS (teal square). 
a
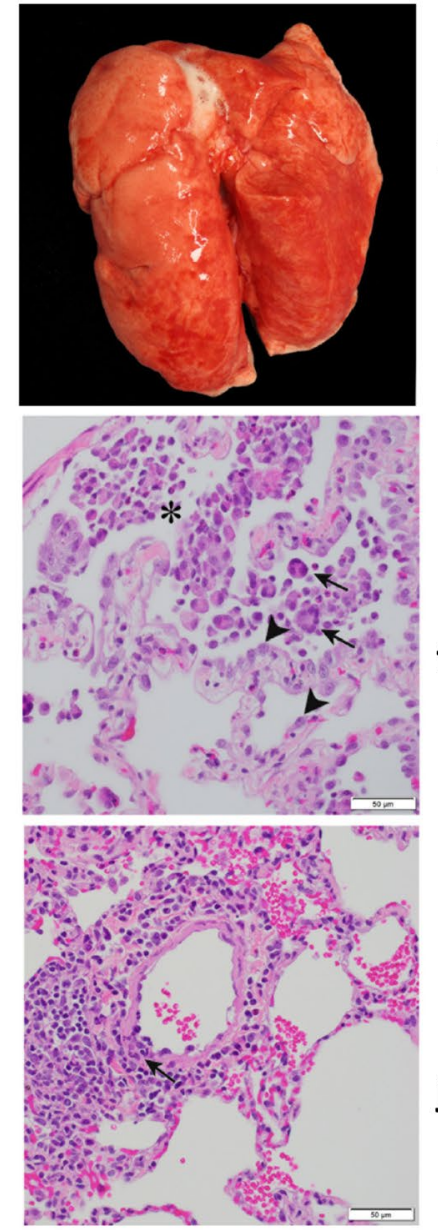
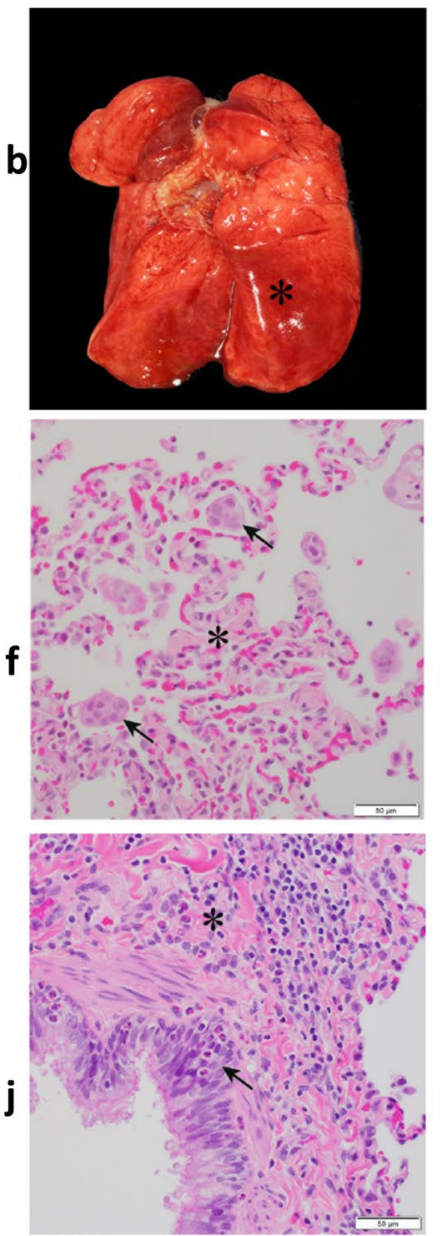
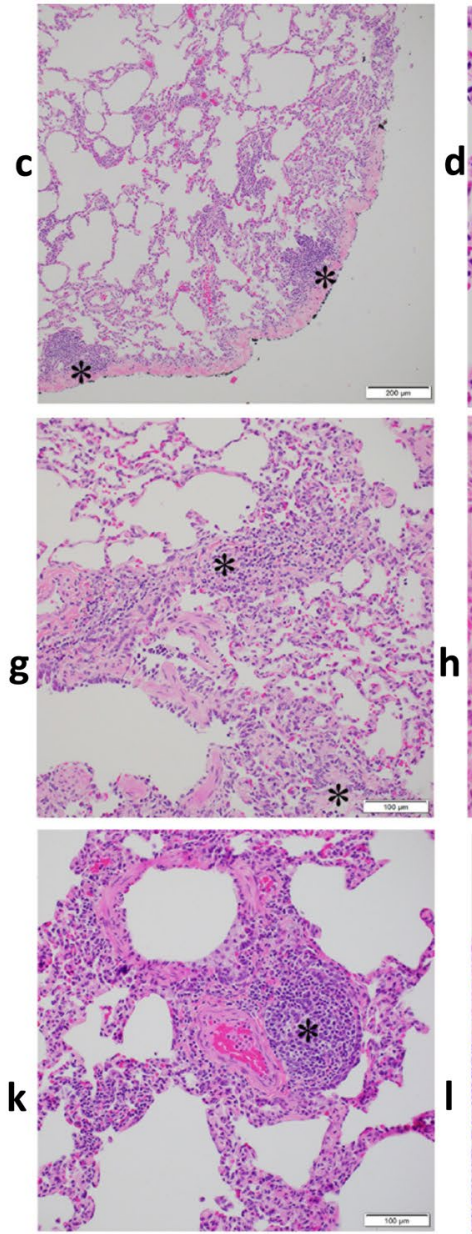
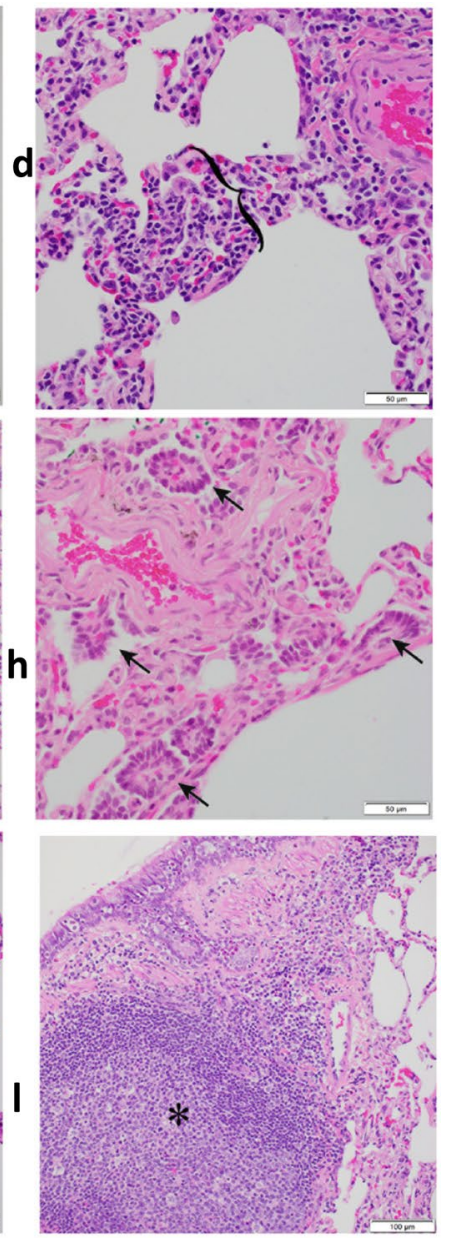

Extended Data Fig. 2 | Gross and histopathologic findings of young and aged male and female Rhesus macaques experimentally exposed to

SARS-CoV-2-14-17 dpi. Young male Rhesus macaque. Lung was grossly unremarkable (a). Aged male Rhesus macaque. The dorsal aspect of the lungs was mottled red (b). Young male Rhesus macaque. Lung. Subgross image showing multifocal areas of minimal interstitial pneumonia $\left({ }^{\star}\right)(\mathbf{c})$. Young female Rhesus macaque. Lung. Mild lymphocytic interstitial pneumonia with alveolar septa (bracket) expanded by mononuclear cells (lymphocytes and macrophages) (d). Aged female Rhesus macaque. Lung. Mild lymphocytic interstitial pneumonia with increased alveolar macrophages and few syncytial cells (arrow) within the alveolar lumen ( ${ }^{*}$; a neutrophil is just to the left of the *) and type II pneumocytes lining alveoli (arrowhead) (e). Aged female Rhesus macaque. Lung. Minimal interstitial pneumonia with alveolar septa expanded by fibrosis $\left({ }^{\star}\right)$ and few syncytial cells (arrow) within alveoli (f). Young male Rhesus macaque. Lung. Alveolar septa expanded by fibrosis $\left(^{\star}\right)$ and lymphocyte infiltrates (g). Aged male Rhesus macaque. Lung. Areas of bronchiolization (arrows) (h). Young female Rhesus macaque. Lung. Vasculitis. Vascular wall disrupted by infiltrates of mononuclear cells and lesser neutrophils (arrow) (i). Young female Rhesus macaque. Lung. Bronchitis. Bronchial epithelium infiltrated by eosinophils (arrow). Fibrosis adjacent to bronchus $\left(^{\star}\right)(\mathbf{j})$. Young female Rhesus macaque. Lung. Area of perivascular lymphocyte infiltrates $\left(^{\star}\right)(\mathbf{k})$. Young female Rhesus macaque. Lung. Area of bronchiolar associated lymphoid tissue (BALT) $\left(^{\star}\right)(\mathbf{I})$. All slides were stained with H\&E. Multiple random fields across all sections from all macaques ( $\mathrm{n}=12$, Supplementary Table 2) were analyzed. 
a
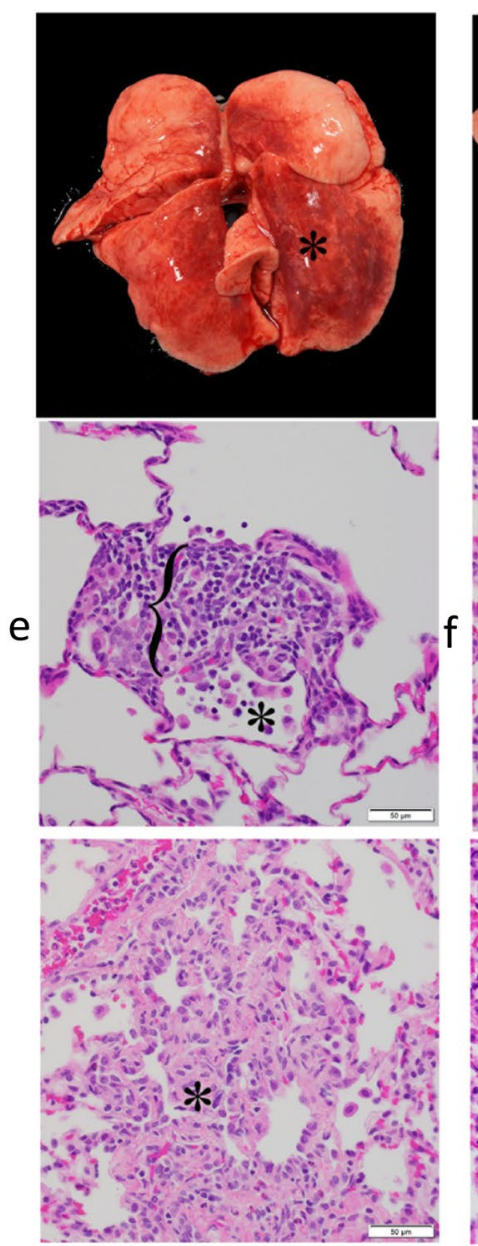

i b
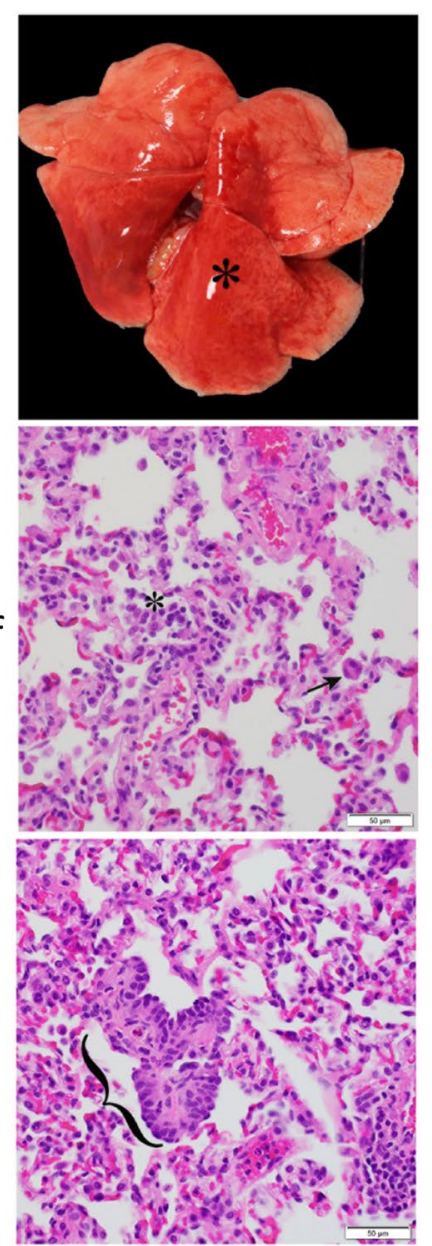

j
C
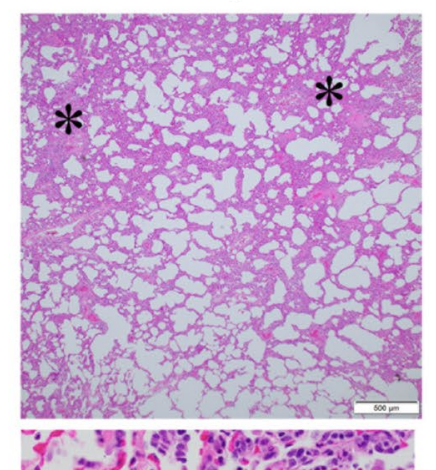

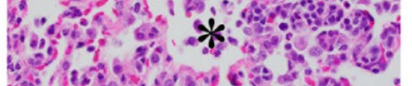

by promesto

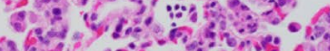
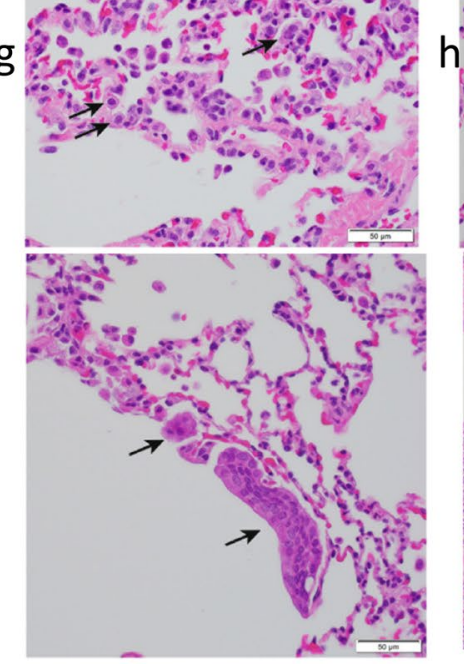

k

d
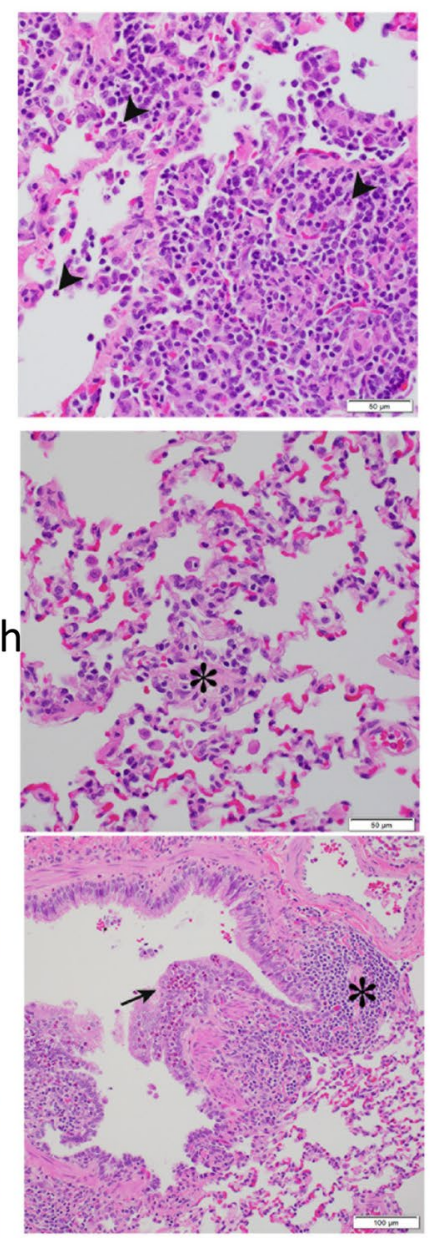

I

Extended Data Fig. 3 | Gross and histopathologic findings of young and aged male and female baboons experimentally exposed to SARS-CoV-2 - 14-17 dpi. Young male baboon. The dorsal aspect of the lungs was mottled red $\left(^{\star}\right)(\mathbf{a})$. Young female baboon. The dorsal aspect of the lungs was mottled red $\left(^{*}\right)$ (b). Young male baboon. Lung. Subgross image showing areas of consolidation $\left(^{\star}\right)$ (c). Young female baboon. Moderate lymphocytic interstitial pneumonia with scattered neutrophils (arrowhead) (d). Young female baboon. Moderate lymphocytic interstitial pneumonia with alveolar septa (bracket) markedly expanded by mononuclear cells (lymphocytes and macrophages) and increased alveolar macrophages within the alveolar lumen $\left(^{\star}\right)(\mathbf{e})$. Young male baboon. Lung. Mild lymphocytic interstitial pneumonia with increased alveolar macrophages and few syncytial cells (arrow) within the alveolar lumen $\left(^{\star}\right)(\mathbf{f})$. Young female baboon. Mild lymphocytic interstitial pneumonia with scattered type II pneumocytes (arrows) and increased alveolar macrophages and neutrophils within the alveolar lumen $\left(^{*}\right)(\mathbf{g})$. Young male baboon. Lung. Alveolar septa expanded by fibrosis $\left(^{\star}\right)(\mathbf{h})$. Young male baboon. Lung. Alveolar septa expanded by fibrosis $\left(^{\star}\right)$ (i). Young female baboon. Area of bronchiolization (bracket) (j). Young male baboon. Lung. Syncytial cells within airways (arrows) (k). Young male baboon. Lung. Bronchitis. Bronchial wall expanded by infiltrates of eosinophils that expand and disrupt the epithelium (arrow). Area of bronchiolar associated lymphoid tissue (BALT) $\left(^{\star}\right)(\mathbf{I})$. All slides were stained with H\&E. Multiple random fields across all sections from all baboons $(n=12$, Supplementary Table 3) were analyzed. 

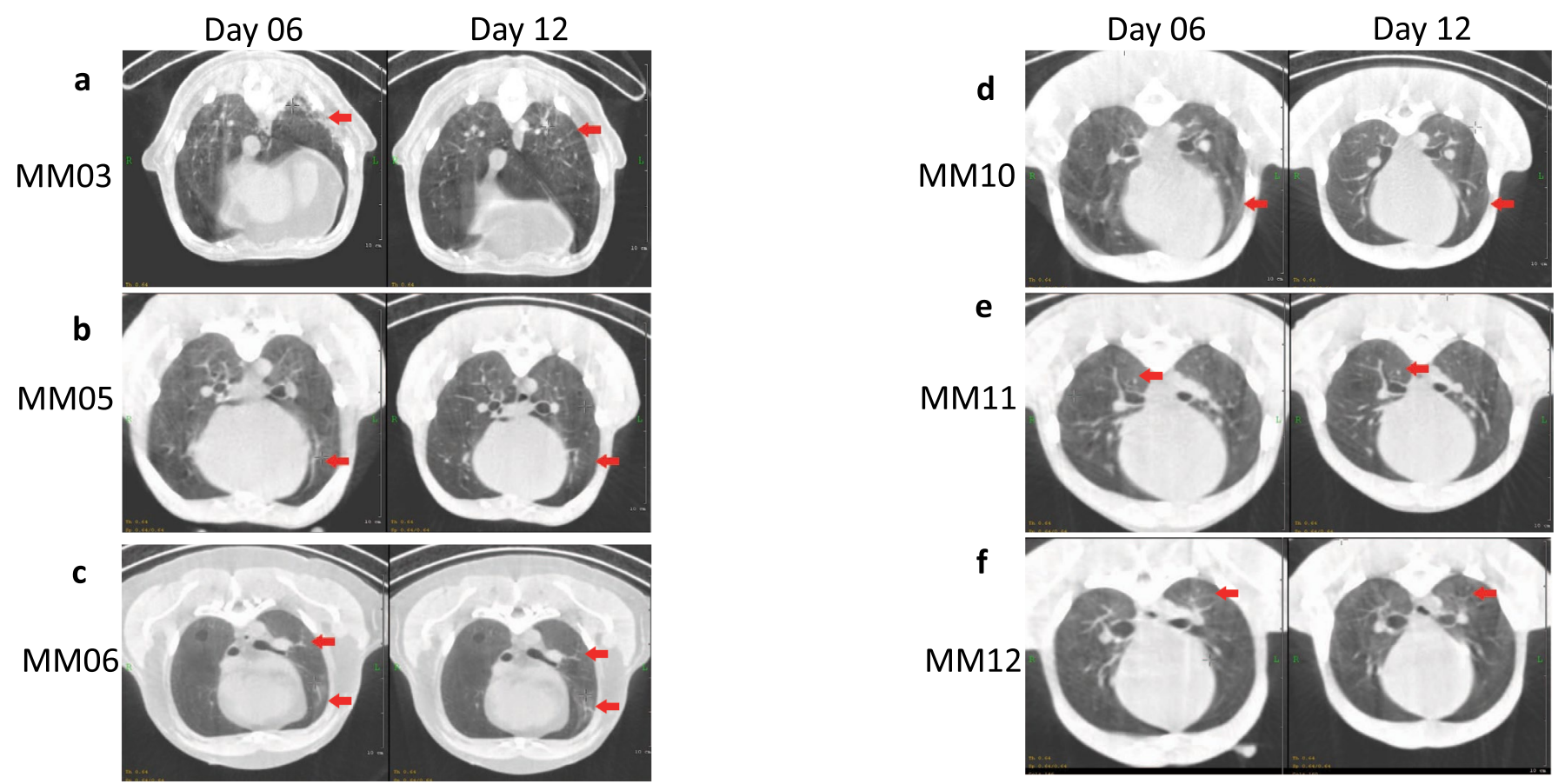

Extended Data Fig. 4 | Representative CT scan images for rhesus macaques infected with SARS-CoV-2 over two weeks. Representative CT scan in axial view showing lesion characteristics in rhesus macaques infected with SARS-CoV-2 from Day 6-12 dpi. As seen in panel $\mathbf{a}, \mathbf{b}, \mathbf{d}, \mathbf{e}$ and $\mathbf{f}$ patchy alveolar patterns, nodular and/or multifocal ground glass opacities (red arrow) seen on Day 6 dpi show dramatic resolution by Day 12 dpi, whereas panel c shows persistent patchy ground glass opacity on Day 6 dpi and Day 12 dpi. 
0.054

a
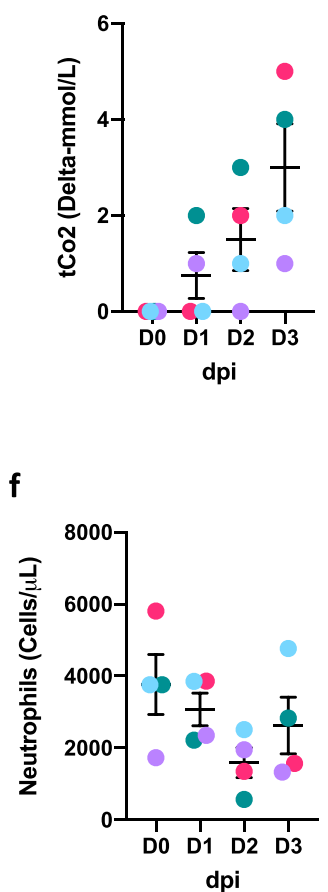

0.0402

b

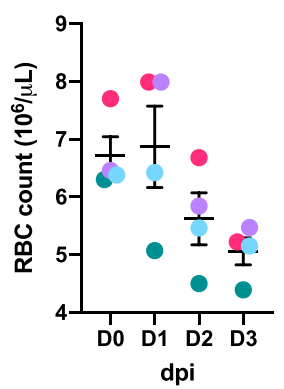

g

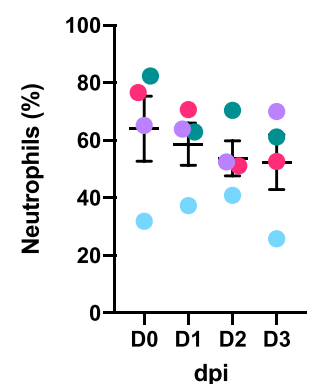

C

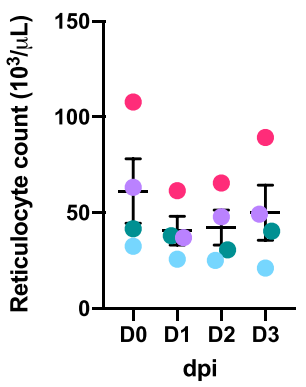

h

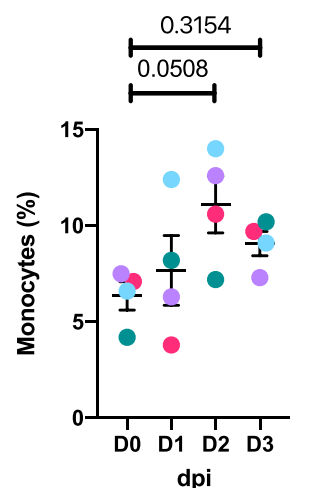

d

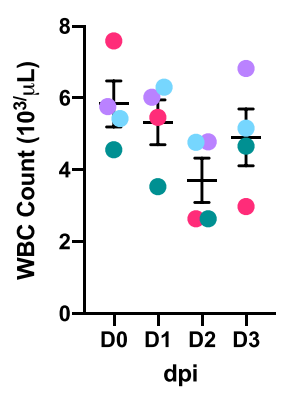

i

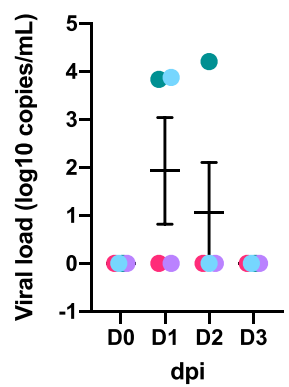

e

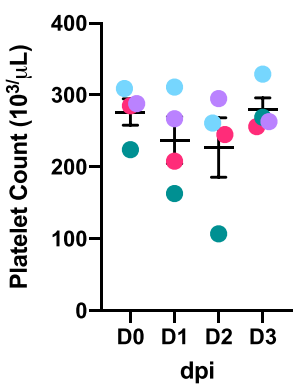

j

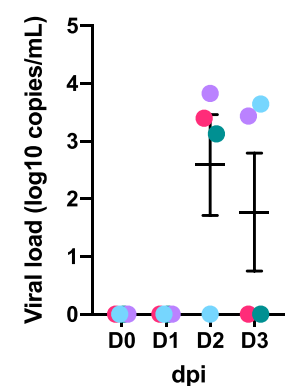

Extended Data Fig. 5 | Clinical correlates in short-term (0-3 dpi) rhesus macaques. Serum levels of tCO2 (D-mmol/L) (a), and whole blood levels of Red Blood Cells $(R B C s)($ million $/ \mu \mathrm{L})(\mathbf{b})$, reticulocytes $(K / \mu \mathrm{L})(\mathbf{c})$, white blood cells $(W B C s)(K / \mu \mathrm{L})(\mathbf{d})$, platelets $(\mathrm{K} / \mathrm{uL})(\mathbf{e}), N$ eutrophils $(\mathrm{cells} / \mu \mathrm{L})(\mathbf{f})$, percentage of Neutrophils $(\mathbf{g})$, percentage of monocytes $(\mathbf{h})$. Viral RNA $\left(\log _{10}\right.$ copies $/ \mathrm{mL}$ were measured by RT-PCR in saliva (i), and rectal swab (j) of rhesus macaques over 0-3 dpi (Circles, Colors represent individual animals, Supplementary Table 1) Data are represented as mean+ SEM ( $n=4$ ). One way Repeated-measures ANOVA with Geisser-Greenhouse correction for sphericity and Tukey's post hoc correction for multiple-testing (GraphPad Prism 8 ) was applied. 


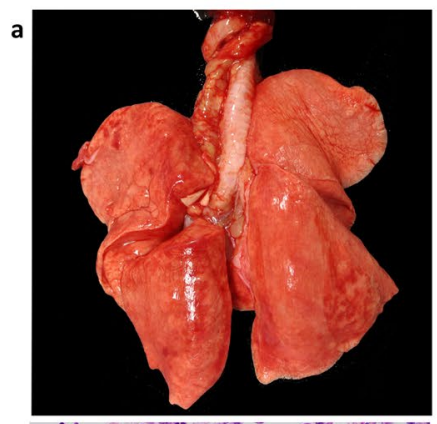

e
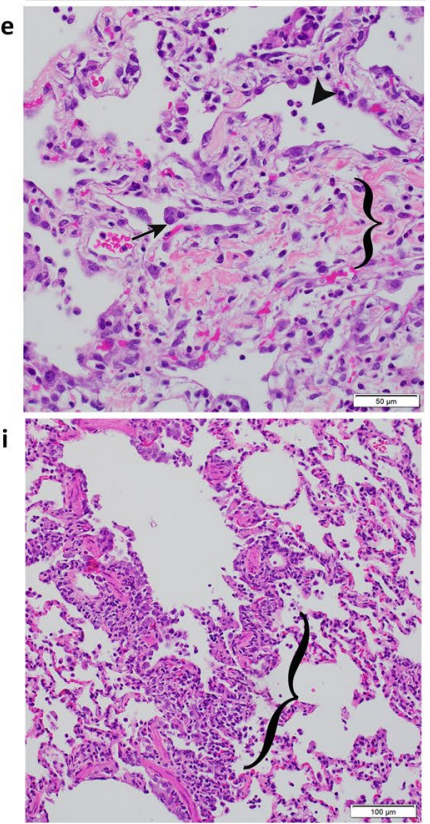
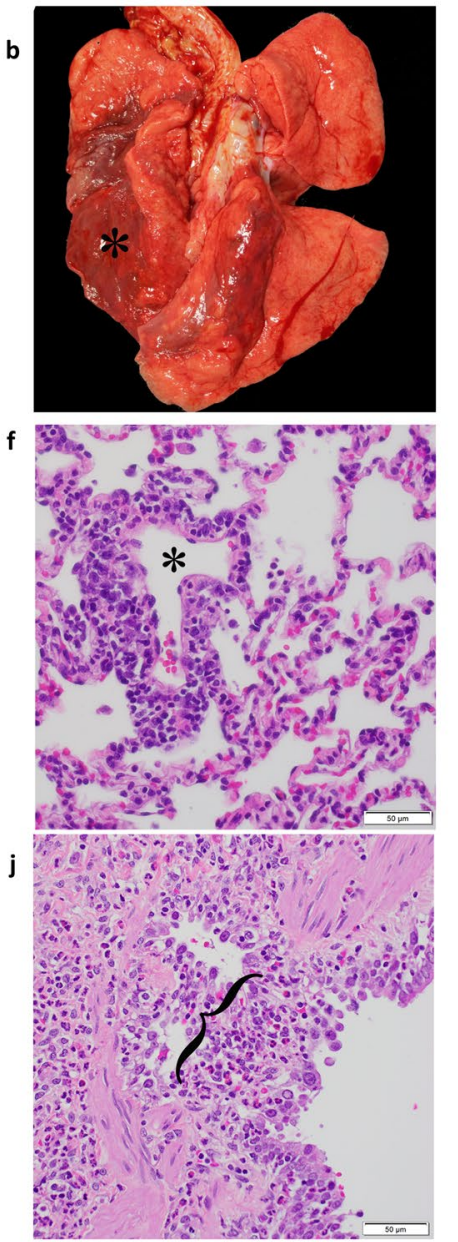
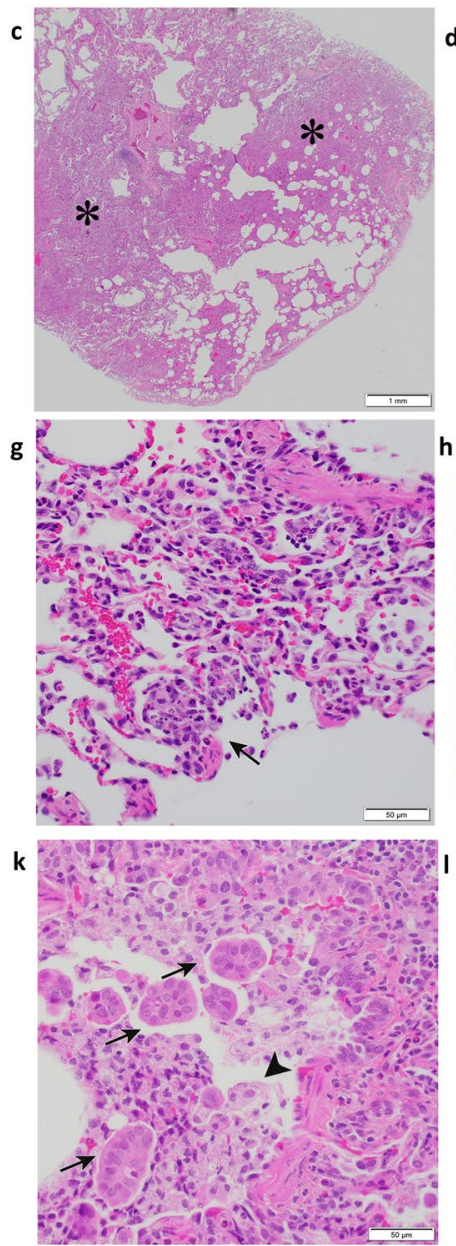
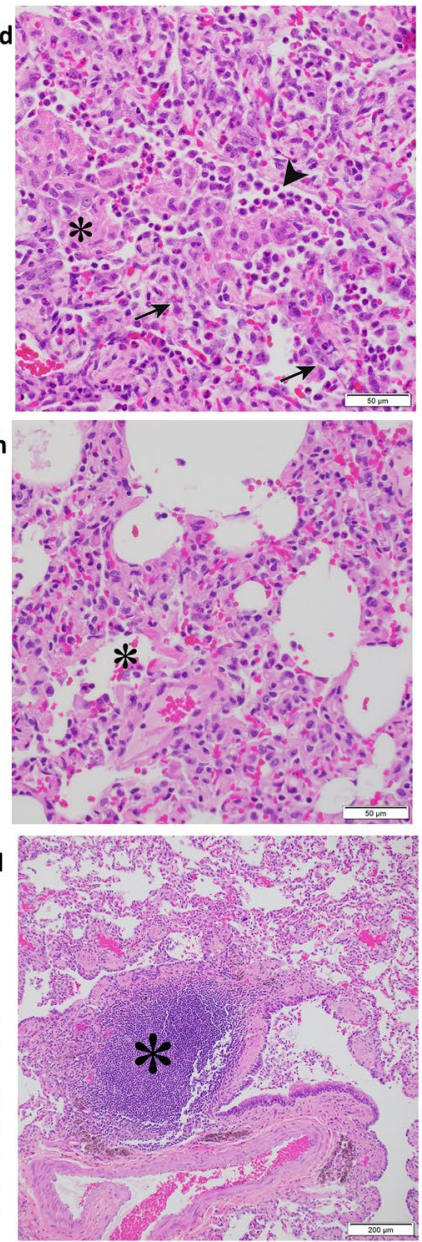

Extended Data Fig. 6 | Gross and histopathologic findings of young and aged male and female Rhesus macaques experimentally exposed to COVID19 - 3 dpi. Young male Rhesus macaque. Lung was grossly unremarkable (a). Aged male Rhesus macaque. Lung. The dorsal aspect of the lungs was mottled $\operatorname{red}\left(^{\star}\right)(\mathbf{b})$. Aged male Rhesus macaque. Lung. Sub gross image showing extensive areas of consolidation $\left(^{\star}\right)$ (c). Aged male Rhesus macaque. Lung. Moderate interstitial pneumonia with scattered type II pneumocytes (arrow), neutrophils (arrowhead), and intra-alveolar fibrin deposition ( ${ }^{\star}$ ) (d). Aged female Rhesus macaque. Lung. Mild interstitial pneumonia with scattered syncytial cells (arrow), neutrophils (arrowhead), and expansion of alveolar walls by fibrosis (bracket) (e). Young female Rhesus macaque. Lung. Vasculitis. Vascular wall disrupted by infiltrates of mononuclear cells and lesser neutrophils. Vessel lumen marked by $\left(^{\star}\right)(\mathbf{f})$. Young female Rhesus macaque. Lung. Mild interstitial pneumonia. Alveolar spaces contain neutrophils and cellular debris (necrosis, arrow) (g). Young female Rhesus macaque. Lung. Mild interstitial pneumonia. Alveolar spaces $\left(^{\star}\right)$ contain neutrophils and eosinophilic fluid (edema) (h). Young female Rhesus macaque. Lung. Bronchiolitis. Bronchiolar wall expanded by infiltrates of lymphocytes and macrophages (bracket) (i). Young male Rhesus macaque. Lung. Bronchitis. Bronchial wall expanded by infiltrates of eosinophils that expand and disrupt the epithelium and smooth muscle (bracket) (j). Young female Rhesus macaque. Lung. Bronchitis. Bronchial lumen contains macrophages (arrowhead), cellular debris, and syncytial cells (arrow) (k). Aged female Rhesus macaque. Lung. Area of bronchiolar associated lymphoid tissue (BALT) ( ${ }^{*}$ ) (I). All slides were stained with H\&E. Multiple random fields across all sections from all macaques ( $n=4$, Supplementary Table 7 ) were analyzed. 
a
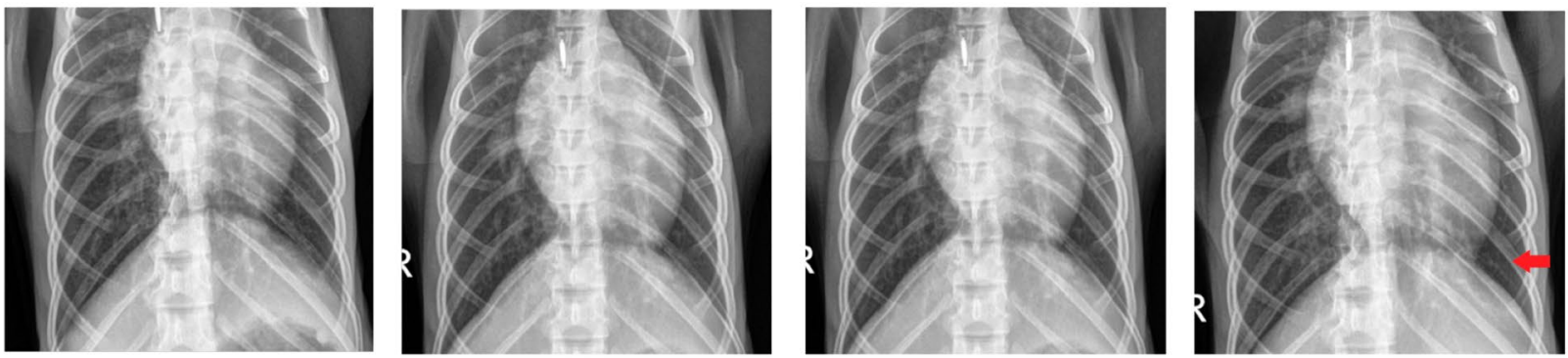

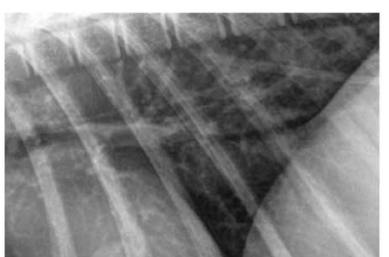

Day 0

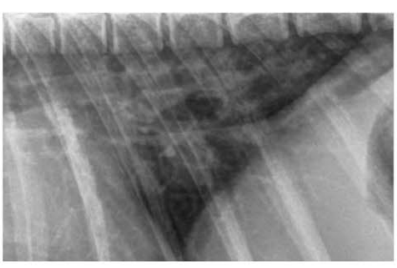

Day 1

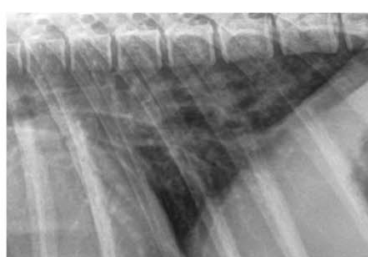

Day 2

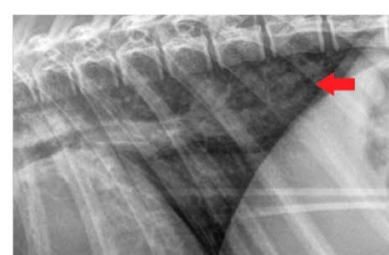

Day 3
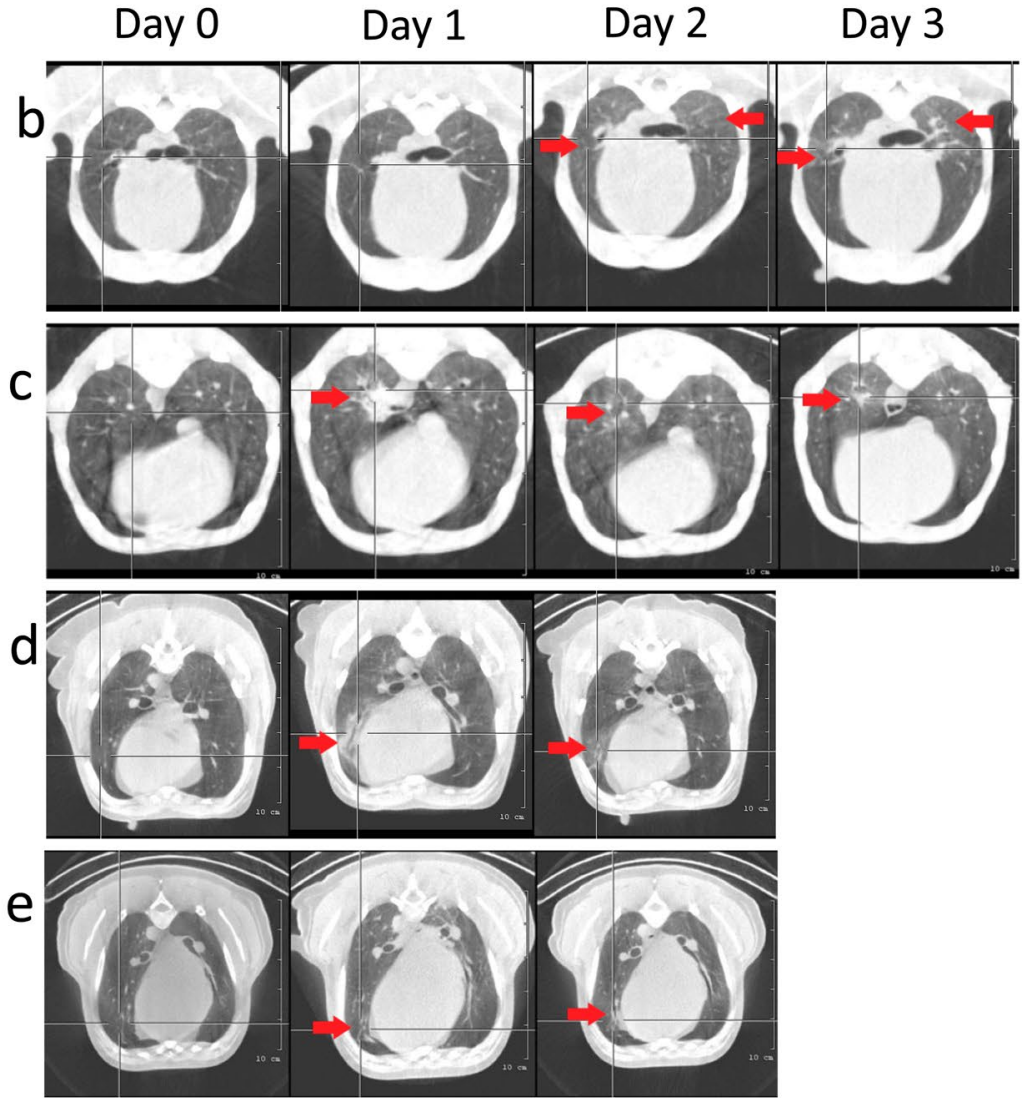

Extended Data Fig. 7 | Radiology of Rhesus macaques experimentally exposed to COVID19 - 3 dpi. CXR Radiographs showing ventro-dorsal and right lateral views(a). Day 0: Normal, Day 1: Mild left caudal interstitial opacity with minimal diffuse right interstitial opacity, Day 2: Mild multifocal interstitial pattern (red arrow), Day 3: Mild multifocal interstitial pattern with patchy region in left caudal lobe (red arrow). CT scan axial view showing lesion characteristics in rhesus macaques infected with SARS-CoV-2 (b) at baseline and Day 1-3 dpi. As seen in (b) ground glass opacity seen on Day 2 dpi intensified on Day 3 dpi. (c) and (d) show lesions that appear on Day 1 show gradual resolution on Day 2-3 dpi whereas lesion in panel (e) observed on Day 1 dpi showed only minimal changes on Day 2. Red arrow point towards lung lesions with high attenuation. 

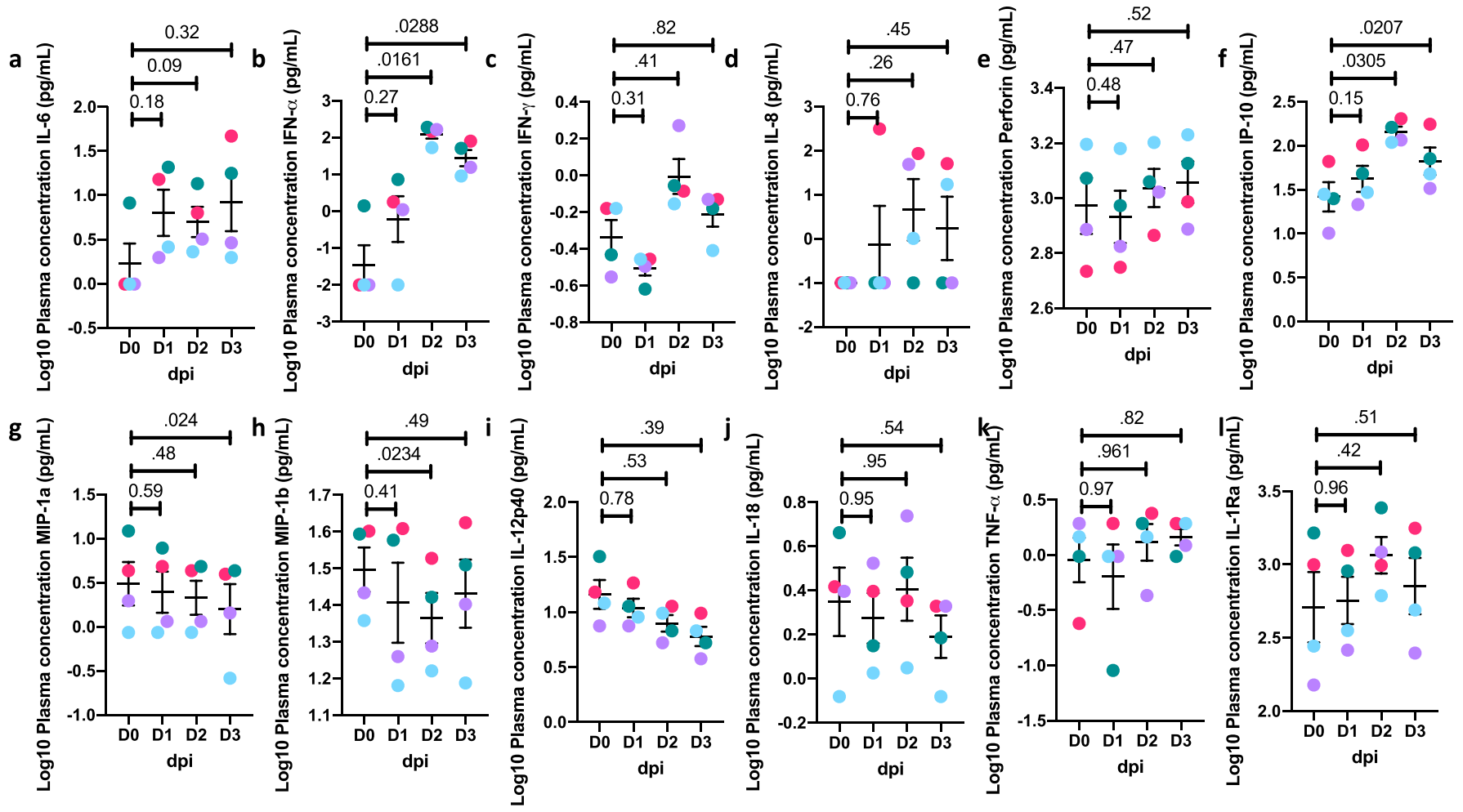

Extended Data Fig. 8 | SARS-CoV-2 induced cytokines in plasma. Simultaneous analysis of multiple cytokines by Luminex technology in the plasma of rhesus macaques over 0-3 dpi. Levels of IL-6 (a), IFN- $\alpha(\mathbf{b})$, IFN- $\gamma(\mathbf{c})$, IL-8 (d), perforin (e), IP-10 (f), MIP1a (g), MIP1b (h), IL-12p40 (i), IL-18 (j), TNF- $\alpha(\mathbf{k})$ and IL-1Ra (I) are expressed in Log10 concentration in picogram per $\mathrm{mL}$ of plasma for rhesus macaques over 0-3 dpi (Circles, Colors represent individual animals, Supplementary Table 1). $(n=4)$ Data are represented as mean+ SEM. One way repeated-measures ANOVA with Geisser-Greenhouse correction for sphericity and Tukey's post hoc correction for multiple-testing (GraphPad Prism 8) was applied. 

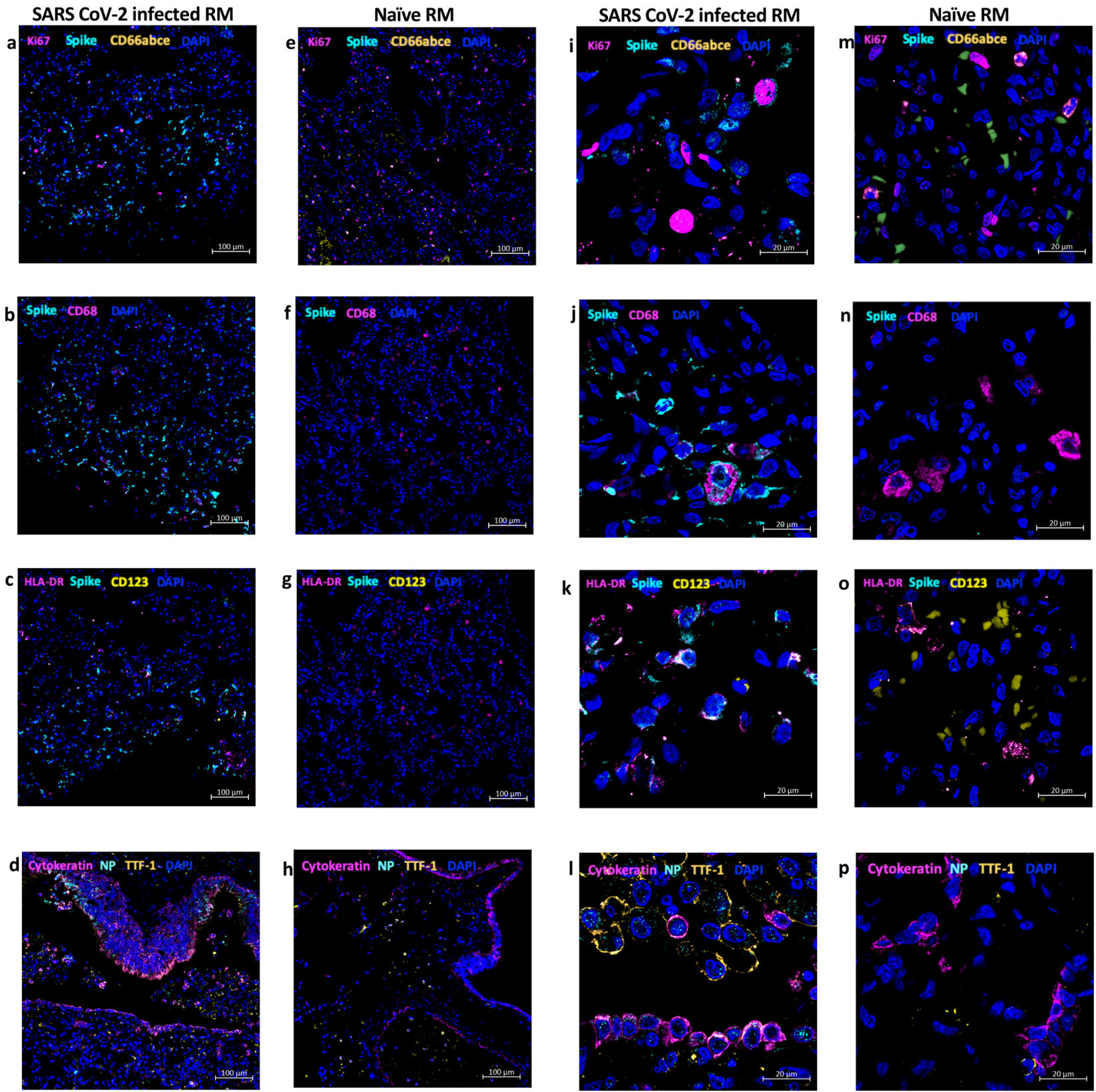

Extended Data Fig. 9 | Detection of SARS-CoV-2 signal in host lung cells by confocal microscopy. Multilabel confocal immunofluorescence microscopy of a high viral titer lung lobe from SARS CoV-2 infected Rhesus macaque at 3 dpi with SARS CoV-2 Spike specific antibody (turquoise), Ki67 (magenta), neutrophil marker CD66abce (yellow) and DAPI (blue)- (10X-a, 63X-i) vs the naïve control lungs (10X-e, 63X-m). SARS CoV-2 Spike (turquoise), pan-macrophage marker CD68 (magenta) and DAPI (blue) in infected lungs (10X-b and 63X-j) vs the naïve control lungs (10X-f, 63X-n). SARS CoV-2 Spike (turquoise), HLA-DR (magenta), pDC marker CD123 (yellow) and DAPI (blue) specific staining in infected lungs (10X-c, 63X-k) vs naïve control lungs (10X-g, 63X-o). SARS CoV-2 Nucleocapsid (turquoise), Type-1 pneumocytes and epithelial marker pan-cytokeratin (magenta), Type-2 pneumocyte marker TTF-1 (yellow) and DAPI (blue) in infected lungs (10X-d, 63X-I) vs naïve control lungs (10X-h, 63X-p). Micrographs are representative of 6 random fields across 3 animals. 

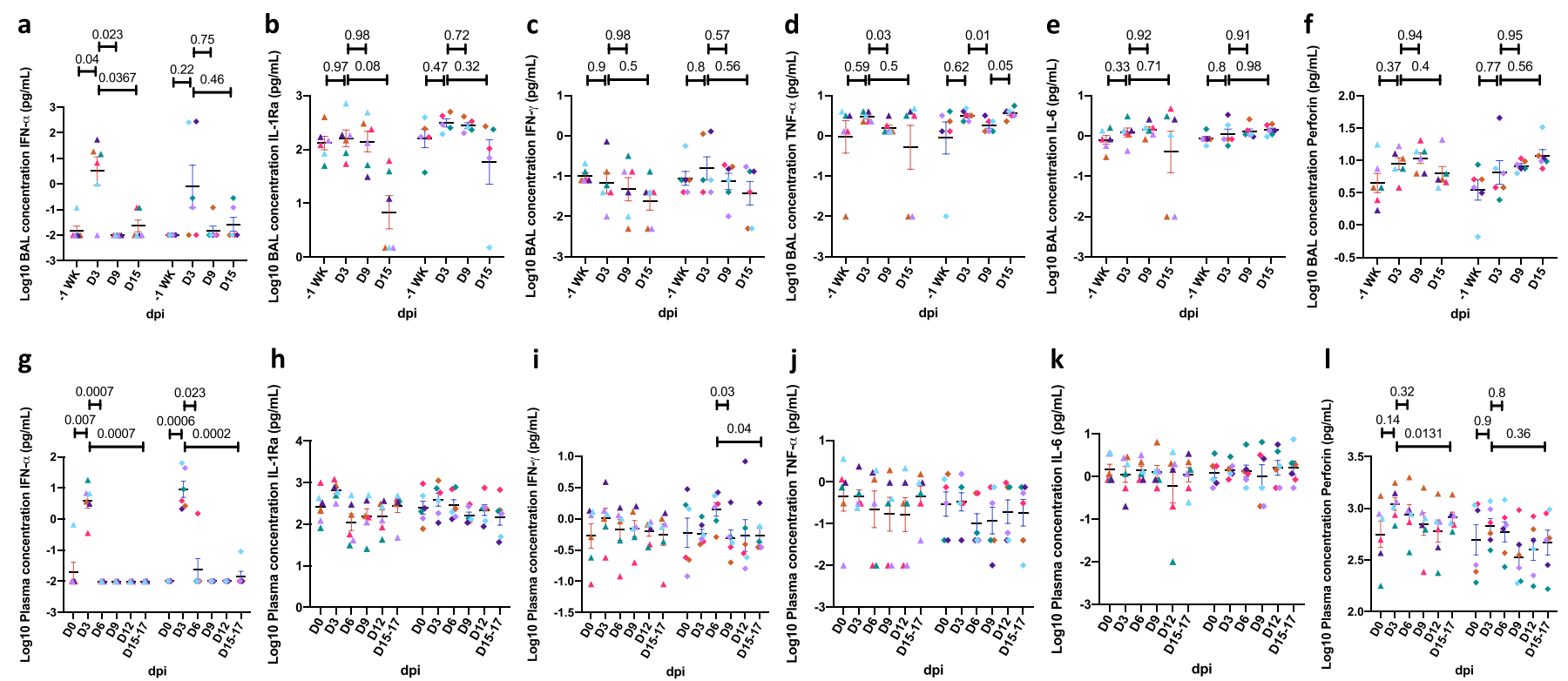

Extended Data Fig. 10 | Longitudinal changes in SARS-CoV-2 induced cytokines in BAL fluid and plasma following SARS-CoV-2 infection in rhesus macaques over two weeks. Simultaneous analysis of multiple cytokines by Luminex technology in the BAL fluid and plasma of rhesus macaques over 0-15 dpi. Levels of IFN- $\alpha(\mathbf{a})$, IL-1Ra (b), IFN- $\gamma(\mathbf{c})$, TNF- $\alpha(\mathbf{d})$, IL-6 (e), Perforin (f) are expressed in Log10 concentration in picogram per mL of BAL fluid. Levels of IFN- $\alpha(\mathbf{g})$, IL-1Ra (h), IFN- $\gamma(\mathbf{i})$, TNF- $\alpha(\mathbf{j})$, IL-6 (k), Perforin (I) are expressed in Log10 concentration in picogram per mL of plasma fluid. Coloring scheme - young (blue), old (red). Data are represented as mean+SEM. $(n=12)$ Two way Repeated-measures ANOVA with Geisser-Greenhouse correction for sphericity and Tukey's post hoc correction for multiple-testing (GraphPad Prism 8) was applied. (Rhesus macaques: Old-Triangle, Young Diamonds, Colors represent individual animals, Supplementary Table 1). 


\section{Reporting Summary}

Nature Research wishes to improve the reproducibility of the work that we publish. This form provides structure for consistency and transparency in reporting. For further information on Nature Research policies, see our Editorial Policies and the Editorial Policy Checklist.

\section{Statistics}

For all statistical analyses, confirm that the following items are present in the figure legend, table legend, main text, or Methods section.

n/a Confirmed

\The exact sample size $(n)$ for each experimental group/condition, given as a discrete number and unit of measurement

$\square$ \ A statement on whether measurements were taken from distinct samples or whether the same sample was measured repeatedly

$\square$ The statistical test(s) used AND whether they are one- or two-sided

$\square$ Only common tests should be described solely by name; describe more complex techniques in the Methods section.

Х $\square$ A description of all covariates tested

$\square$ \A description of any assumptions or corrections, such as tests of normality and adjustment for multiple comparisons

$\square$ A full description of the statistical parameters including central tendency (e.g. means) or other basic estimates (e.g. regression coefficient)

$\bigotimes$ AND variation (e.g. standard deviation) or associated estimates of uncertainty (e.g. confidence intervals)

$\varnothing$ For null hypothesis testing, the test statistic (e.g. $F, t, r$ ) with confidence intervals, effect sizes, degrees of freedom and $P$ value noted

Give P values as exact values whenever suitable.

Х $\square$ For Bayesian analysis, information on the choice of priors and Markov chain Monte Carlo settings

Х $\square$ For hierarchical and complex designs, identification of the appropriate level for tests and full reporting of outcomes

$\bigotimes$ Estimates of effect sizes (e.g. Cohen's $d$, Pearson's $r$ ), indicating how they were calculated

Our web collection on statistics for biologists contains articles on many of the points above.

\section{Software and code}

Policy information about availability of computer code

Data collection BD FACSDiva Software v8.0.1

SmartDR

VetScan VS2 Analyzer 0000V25158

IDEXX VetLab Station IPU 00-34 Build 57

QuantStudio Design and Analysis Software v1.4.3

Olympus cellSens Entry Imaging Software Version 1.18

Nucline nanoScan LFER 1.07.004.0000

ZEN Imaging software version 2.1 (blue edition)

XPONENT V3.1 or 4.3

Data analysis Flowjo v10.6.2

SmartPacs

VetScan VS2 Analyzer Abaxis 0000V25158

IDEXX VetLab Station IPU 00-34 Build 57

QuantStudio Design and Analysis Software v1.4.3

Olympus cellsens Entry Imaging Software Version 1.18

Vivoquant 4.0 patch 3

ZEN Imaging software version 2.1 (blue edition)

Merck Belysa 1.0.19 
Policy information about availability of data

All manuscripts must include a data availability statement. This statement should provide the following information, where applicable:

- Accession codes, unique identifiers, or web links for publicly available datasets

- A list of figures that have associated raw data

- A description of any restrictions on data availability

All the data that support the findings of this study are available within the current manuscript and associated supplement. Any additional data can be requested from the corresponding authors upon reasonable request.

\section{Field-specific reporting}

Please select the one below that is the best fit for your research. If you are not sure, read the appropriate sections before making your selection. $\bigotimes$ Life sciences $\square$ Behavioural \& social sciences Ecological, evolutionary \& environmental sciences

For a reference copy of the document with all sections, see nature.com/documents/nr-reporting-summary-flat.pdf

\section{Life sciences study design}

All studies must disclose on these points even when the disclosure is negative.

Sample size Since this was a model establishment study for COVID, and due to non existent data available on NHPs the sample sizes were determined based on our prior experience on infectious disease research.

Data exclusions No data were excluded.

Replication All attempts at replication were successful. Flow cytometry: 1 sample per subject at each timepoint, $\mathrm{n}=12$ Confocal analysis: 6 random fields per subject, $n=3$

Pathology: $>8$ random fields per subject, $\mathrm{n}=12$

Virology: Duplicate

Multiplex Cytokine Analysis: Duplicate

Randomization Individual animals were randomly assigned to different groups.

Blinding Support staff, technicians and investigators only involved in data collection were blinded to study groups. Due to involvement of some investigators in animal monitoring and managing the experiments throughout the study, complete blinding was not possible. Few of these investigators were involved in analysis and completely blinded data analysis was not achieved.

\section{Reporting for specific materials, systems and methods}

We require information from authors about some types of materials, experimental systems and methods used in many studies. Here, indicate whether each material, system or method listed is relevant to your study. If you are not sure if a list item applies to your research, read the appropriate section before selecting a response.

Materials \& experimental systems

\begin{tabular}{|c|c|}
\hline $\mathrm{n} / \mathrm{a}$ & Involved in the study \\
\hline & $\bigotimes$ Antibodies \\
\hline & $\bigotimes$ Eukaryotic cell lines \\
\hline & $\square$ Palaeontology and archaeology \\
\hline & $\bigotimes$ Animals and other organisms \\
\hline 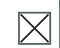 & $\square$ Human research participants \\
\hline - & $\square$ Clinical data \\
\hline$\nabla$ & $\square$ Dual use research of concern \\
\hline
\end{tabular}

Methods

\begin{tabular}{l|l}
\hline n/a & Involved in the study \\
$\square$ & $\square$ ChIP-seq \\
$\square$ & $\bigotimes$ Flow cytometry \\
$\square$ & $\square$ MRI-based neuroimaging
\end{tabular}

\section{Antibodies}

Antibodies used

Validation
A description of all antibodies used is provided as Supplementary Table 8.

A description of validation statement for all antibodies used is provided as Supplementary Table 8. 
Policy information about cell lines

Cell line source(s)

ATCC

Authentication

Acquired from ATCC with Certificate of authentication

Mycoplasma contamination

Commonly misidentified lines

(See ICLAC register)

Negetive

None

\section{Animals and other organisms}

Policy information about studies involving animals; ARRIVE guidelines recommended for reporting animal research
Laboratory animals
Details for all the animals is attaches as Supplementary Table 1.

Wild animals

No wild animals were used in the study

Field-collected samples

No field collected samples were used in the study.

Ethics oversight

The animal studies in each of the species were approved by the Animal Care and Use Committee of the Texas Biomedical Research Institute and as an omnibus Biosafety Committee protocol.

Note that full information on the approval of the study protocol must also be provided in the manuscript.

\section{Flow Cytometry}

\section{Plots}

Confirm that:

\The axis labels state the marker and fluorochrome used (e.g. CD4-FITC).

\The axis scales are clearly visible. Include numbers along axes only for bottom left plot of group (a 'group' is an analysis of identical markers).

\All plots are contour plots with outliers or pseudocolor plots.

\A numerical value for number of cells or percentage (with statistics) is provided.

\section{Methodology}

Sample preparation

Instrument

Software

Cell population abundance

Gating strategy

Xick this box to confirm that a figure exemplifying the gating strategy is provided in the Supplementary Information.
Cells were analyzed using a FACSymphony A3 flow cytometer (BD Biosciences).

BD FACSDiva Software v8.0.1

No sorting was done in this study.

The gating strategy is included as Supplementary Figure 6.
All details pertaining to sample preparation are included or cited in text and methods section. 